\title{
Stress-induced cross-feeding of internal metabolites provides a dynamic mechanism of microbial cooperation
}

\author{
Kapil Amarnath ${ }^{1}$, Avaneesh V. Narla ${ }^{1, \dagger}$, Sammy Pontrelli ${ }^{2, \dagger}$, Jiajia Dong ${ }^{1,3, \dagger}$, Tolga Caglar ${ }^{1}$, Brian R. \\ Taylor $^{1}$, Julia Schwartzman ${ }^{4}$, Uwe Sauer ${ }^{2}$, Otto X. Cordero ${ }^{4}$, Terence Hwa ${ }^{1, *}$ \\ ${ }^{1}$ Department of Physics, U.C. San Diego, La Jolla, CA 92093-0319 \\ ${ }^{2}$ Institute of Molecular and Systems Biology, ETH Zürich, Switzerland \\ ${ }^{3}$ Department of Physics and Astronomy, Bucknell University, Lewisburg, PA 17837 \\ ${ }^{4}$ Department of Civil and Environmental Engineering, MIT, Cambridge, MA 02139 \\ $\dagger$ equal contribution \\ * corresponding author: Terence Hwa (thwa@ucsd.edu)
}

\begin{abstract}
Despite the ubiquity of microbial diversity observed across environments, mechanisms of cooperativity that enable species coexistence beyond the classical limit of one-species-per-niche have been elusive. Here we report the observation of a transient but substantial cross-feeding of internal metabolites between two marine bacterial species under acid stress, and further establish through quantitative physiological characterization of the individual strains that this cross-feeding is central to the survival and coexistence of these species in growth-dilution cycles. The coculture self-organizes into a limit cycle in which acid-stressed producers excrete various internal metabolites upon entering growth arrest, enabling the cross-feeders to grow, restore medium $\mathrm{pH}$, and protect the producers from death. These results establish a rare, mechanistic example of inter-species cooperation under stress, as anticipated long ago by the stress gradient hypothesis. As the accumulation of acetate and other fermentation products occurs ubiquitously in habitats ranging from the gut to wastewater, and the excretion of internal metabolites is an obligatory physiological response by bacteria under weak acid stress, stressinduced cross-feeding provides a general physiological basis for the extensive sharing of metabolic resources to promote the coexistence of diverse species in microbial communities.
\end{abstract}

Metabolic cross-feeding, or syntrophy, underlies many positive interactions between microbes ${ }^{1-3}$. Recently, extensive cross-feeding of internal metabolites was proposed as a general mechanism ${ }^{4-6}$ for microbial communities to maintain diversity well beyond the limit imposed by classical competition theories ${ }^{7-9}$. Although the excretion of metabolites $h ß a s$ been intensely studied for waste products of anaerobic digesters ${ }^{10-}$ ${ }^{13}$, cross-feeding of central metabolites such as carbon precursors, co-factors, and amino acids at flux levels needed to support substantial growth of other species is physiologically costly and is only known anecdotally for closely associated symbionts ${ }^{14-16}$ or for synthetic strains and conditions ${ }^{17-25}$.

In principle, the origin(s) of microbial cooperativity can be elucidated by analyzing natural communities that support many bacterial species when fed with a few nutrient sources ${ }^{4,26-29}$. However, the inherent complexity of such communities precludes the detailed studies necessary to reveal mechanisms underlying coexistence. In our study, we focus exclusively on the interaction between a pair of bacterial species from such a community. A novel mechanism of cooperativity is revealed by characterizing how these bacteria turn from competitors to collaborators. 


\section{Results}

\section{Acetate and ammonia cross-feeding between two co-isolated bacteria}

Vibrio splendidus sp. 1A01 and Neptunomonas phytotrophica $\mathrm{sp}$. 3B05 are two species co-isolated from a chitin enrichment culture of coastal ocean water ${ }^{30}$. When cultured alone on chitin, 1A01 grew but 3B05 did not (Extended Data Fig. 1a). To investigate possible reasons for the presence of 3B05 and many other nonchitin-degrading bacteria in the enrichment culture ${ }^{30}$, we grew these two strains together using $\mathrm{N}$-acetylglucosamine (GlcNAc), the monomer of chitin, as the sole carbon and nitrogen source, in defined medium strongly buffered at $\mathrm{pH}=8$, the nominal $\mathrm{pH}$ of sea water ${ }^{31}$; see Materials and Methods. After inoculating $1 \mathrm{~A} 01$ and 3B05 at equal ratio, the coculture was left to grow for 24 hours, then diluted 40-fold into fresh medium. Such 24-h growth-dilution cycles were repeated for several days (Fig. 1a). Before each dilution, the abundance of each species was monitored using 16S PCR (Extended Data Fig. 1b) ${ }^{32}$. The two species were found to coexist stably, settling after a few cycles to a ratio 3B05:1A01 1:3 by 16S abundance (Fig. 1b, black diamonds).

To determine the mechanistic basis of coexistence, we quantified the growth and uptake/excretion characteristics of each species in monoculture. Only 1A01 grew in monoculture with GlcNAc as the sole carbon/nitrogen source (Extended Data Fig. 1c). We analyzed the culture medium by HPLC and found substantial accumulation of acetate and ammonium (Extended Data Fig. 1d). A closer examination of the excretion data suggests that the acetate liberated in the conversion of GlcNAc to glucosamine ${ }^{33}$ was directly released into the medium (Extended Data Fig. 1e).

The substantial excretion of acetate and ammonium by $1 \mathrm{~A} 01$ in monoculture suggested that $3 \mathrm{~B} 05$ might be growing on these carbon and nitrogen sources in the coculture. As a first test, we grew $1 \mathrm{~A} 01$ and 3B05 as monocultures on acetate and ammonium, and found that only 3B05 grew (Extended Data Fig. 1f). The results suggest that a simple commensalism between 1A01 and 3B05 (solid lines, Fig. 1c) underlies the coexistence found in Fig. 1b. Indeed, properties of the coculture can be quantitatively explained by single strain characteristics summarized in Extended Data Table 1; see e.g., Extended Data Fig. 1g.

\section{Acetate cross-feeding insufficient to support coexistence in weak buffer}

One important feature of the coculture described above is the high buffer capacity (40 mM HEPES) used, which fixed the medium $\mathrm{pH}$ and enabled us to focus solely on nutrient consumption and cross-feeding. A scenario of potentially broad ecological relevance is one in which growth in the coculture is limited not only by nutrients but also by medium acidification resulting from acetate excretion, as many natural environments including the ocean have low buffering capacity ${ }^{34-36}$ (dashed line, Fig. 1c). Assuming that 3 B05 is less affected by reduced $\mathrm{pH}$ than 1A01 in a weakly buffered coculture, the growth of 3B05 on acetate would benefit $1 \mathrm{~A} 01$ by removing the source of toxicity and restoring the medium $\mathrm{pH}$, resulting in the exponential growth of the two species at the same rate; see Extended Data Fig. 2a. However, when we measured the $\mathrm{pH}$-dependences of the growth of 1A01 and 3B05 monocultures, we observed that 1A01 always grew faster (Extended Data Fig. 2b). Thus 3B05 is not expected to relieve acidification by the growth of $1 \mathrm{~A} 01$ in weakly buffered media, ruling out the canonical, stable, exponentially growing coculture of the type depicted in Extended Data Fig. 2a ${ }^{37}$. Instead, a coculture of 1A01 and 3B05 should stop growing before the full consumption of the GlcNAc, since the faster growth of 1A01 compared to 3B05 implies that acetate would eventually be generated at a faster rate than it would be consumed (Extended Data Fig. 2c). Indeed, a coculture of 1A01 and 3B05 in $2 \mathrm{mM}$ bicarbonate (the buffer in the ocean, primarily from equilibration with atmospheric $\left.\mathrm{CO}_{2}\right)^{36}$ stopped growing $\sim 6$ hours after inoculation, when the $\mathrm{pH}$ dropped sharply (Extended Data Fig. 2d) before the full consumption of GlcNAc (Extended Data Fig. 2e). The dynamics of the 
coculture observed here is quantitatively captured by a simple metabolic model (Supp Note 1), using singlestrain characteristics obtained from the two monocultures.

Because 3B05's growth rate prevented it from growing 40-fold in 6 hours on acetate/ammonium (Extended Data Fig. 1f), we expected it to be depleted from the coculture if the growth-dilution experiment of Fig. 1a were repeated in the weak buffer. Contrary to our expectation, however, coexistence remained in the 24-h growth-dilution experiments in the weak $2 \mathrm{mM}$ bicarbonate buffer, settling to an even higher stable ratio of 3B05 to 1A01 than that in the strong buffer case (filled green diamonds, Fig. 1b). Moreover, measurements of GlcNAc and acetate concentration in the medium at the end of each cycle showed the complete consumption of GlcNAc with no acetate accumulation once the coculture stabilized after a few cycles (Fig. 1d). To look for possible syntrophic interaction that might have escaped our analysis, we repeated the growthdilution experiment with 6-h cycles to maintain the coculture in exponential growth, mimicking a chemostat (since the coculture grew exponentially the first 6 hours, see Extended Data Fig. 2d). The frequency of 3B05 is seen to decay exponentially (Fig. 1e, open diamonds), i.e., 3B05 is outcompeted by 1A01, as expected. Thus, the coexistence observed in the 24-h growth-dilution experiment resulted from some syntrophic effect that occurred outside of the exponential growth phase ${ }^{38}$.

\section{Cell death and its rescue in the coculture}

To elucidate what occurred in the non-growing phase of the 24-h growth-dilution experiment in weak buffer, we measured the density of viable 1A01 and 3B05 cells by plating the coculture on marine broth plates and distinguishing the two species by colony morphology (Extended Data Fig. 3a-c). The data collected over the first cycle (Extended Data Fig. 3d) shows that beyond exponential growth during the first 6 hours, the count of viable 1A01 cells dropped rapidly, eventually reaching 1000x below its maximum by the time of dilution at 24 hours. During this time, viable 3B05 cells stayed constant. We verified that the death of $1 \mathrm{~A} 01$ was due to medium acidification (Extended Data Fig. 3e). The occurrence of massive death raised a possible question of whether the coexistence seen in 24-h growth-dilution experiment might have resulted from mutation and selection. Repeating this experiment using clones isolated from the end of 5 growth-dilution cycles (open diamonds, Fig. 1b) shows that evolution is not a concern here.

Since acidification of the environment and the subsequent "ecological suicide" have been suggested to play important roles in microbial community dynamics ${ }^{39,40}$, we investigated whether the coexistence observed in our system might have resulted from the altered population dynamics associated with the fast death of $1 \mathrm{~A} 01$ in low $\mathrm{pH}$. We expanded our metabolic model of the coculture to growth-dilution cycles and included the measured characteristics of cell death (Supp Note 2). The model indeed gave rise to coexistence after several cycles, with the main effect of death being a large reduction in the ratio of the viable 1A01 to 3B05 cells at the start of a new growth cycle, thereby giving the slower 3B05 more time to grow and catch up within each cycle (Extended Data Fig. 3f,h). Another key feature of the model with death is the inability of the coculture to fully consume the GlcNAc provided due to acetate accumulation (Extended Data Fig. 3g,i). These predictions are however in clear disagreement with our data: not only was GlcNAc completely consumed after a few cycles (Fig. 1d), the viable cell counts (circles, Fig. 1f) stabilized to similar levels as those in the stress-free case with strong buffer (arrows, Fig. 1f). Thus, cell death was not a key contributor to the stability of the coculture.

\section{Growth and metabolite dynamics in the stable cycle}

Since 1A01 lost viability extremely rapidly upon exposure to acetate in weak buffer (Extended Data Fig. 3d,e) while it maintained viability at a high level several cycles into coculture growth (Fig. 1f), the coculture 
apparently developed a protective effect to hold down acetate accumulation after a few cycles. We thus turned to investigating the nature of this protective effect, by analyzing the simpler dynamics of the coculture in the "stable cycle", several cycles after the initial inoculation (Fig. 1d,f). As we will show later, understanding this protective effect will also allow us to address the approach to the stable cycle from the initial inoculation.

We measured the viability of 1A01 and 3B05 (Fig. 2a) and concentrations of GlcNAc and acetate (Fig. 2b) during the stable cycle and observed strikingly different dynamics from that observed for the first cycle. First, the acetate concentration in the medium peaked only briefly in the middle of the cycle (red squares in Fig. 2b), with all of it consumed shortly after 1A01 stopped growing, which coincided with the depletion of GlcNAc. Compared to the prolonged exposure to high acetate in the first cycle (Extended Data Fig. 2e), we see that the maintenance of $1 \mathrm{~A} 01$ viability can indeed be attributed to acetate removal in the stable cycle. Also, the growth of 3B05 (open black circles, Fig. 2a) surged during the time acetate was depleted (shaded region), even though the coculture including 3B05 stopped growing at high acetate levels in the first cycle (Extended Data Fig. 3d). Second, at the beginning of the stable cycle, 1A01 did not grow despite the removal of acetate and availability of GlcNAc in the fresh medium. (The apparent drop in CFU of 1A01 in the 6-h following dilution was due to a moderate degree of cell clumping, not cell death; see Extended Data Fig. 5.) Equally puzzling, 3B05 managed to grow when $1 \mathrm{~A} 01$ was not growing, even though there was no acetate left over from the previous cycle. This lag in the growth recovery of 1A01 gave 3B05 extra time to "catch up" with the faster growing 1A01 cells. Below we separately investigate factors enabling the surge of 3B05 growth following the arrest of $1 \mathrm{~A} 01$ and the delay in the growth recovery of 1A01 following dilution.

\section{Growth of $3 B 05$ after the arrest of 1 A01 was aided by other internal metabolites}

To determine the cause of the surge in 3B05 towards the end of coculture growth, we densely measured cell viability and analyzed the spent medium during the period when acetate peaked to correlate changes in the medium with changes in the growth of cells (Fig. 2c-f). Analysis of the medium by HPLC revealed that, in addition to acetate, several internal metabolites, namely pyruvate, lactate, and glutamate, accumulated to surprisingly large concentrations starting from 30-60 min before GlcNAc was exhausted (Fig. 2e). The ensuing disappearance of these internal metabolites (grey shaded region) coincided with the depletion of acetate (red squares, Fig. 2d) and the surge of 3B05 growth, while viable counts of 1A01 remained constant during this period (Fig. 2c). During its surge, 3B05 grew at a rate substantially larger than on acetate alone (compare solid and dash-dotted lines in Fig. 2c). This faster growth rate is consistent with 3B05's growth rate on a mixture of acetate, lactate, pyruvate, and glutamate at normal $\mathrm{pH}$ (Extended Data Table 1), suggesting that the surge of 3B05 was aided by the internal metabolites in the medium. The consumption of these internal metabolites in addition to acetate would also account for the higher cell density of $3 \mathrm{~B} 05$ reached in the stable cycle in weak buffer compared to that in strong buffer (Fig. 1f, compare open circles and open arrow).

An equally puzzling aspect of the dynamics was how 3B05 managed to maintain some growth when acetate peaked. To investigate this phenomenon, we grew 3B05 monoculture in acidified medium with and without the supplement of the internal metabolites lactate, pyruvate, and glutamate which accumulated transiently in the coculture. 3B05 only grew with the supplement (Fig. 3a), resulting in acetate uptake (Fig. 3b), and pH recovery (Fig. 3c). Apparently, these supplements relieved enzymatic bottlenecks that inhibited the growth of $3 \mathrm{~B} 05$ on acetate alone.

\section{Internal metabolites were extensively excreted by acetate-stressed 1 A01 cells}


To clarify where these internal metabolites came from, we mimicked the conditions during the acetate peak in the stable cycle of the coculture by growing 1A01 monoculture in GlcNAc while maintaining the $\mathrm{pH}$ between 5-5.3 (Fig. 3d). During this period, GlcNAc was depleted while acetate, lactate, pyruvate, and glutamate accumulated in the medium (Fig. 3e, 3f). Thus, these internal metabolites were excreted by 1 A01 under acetate stress. Quantitatively, the depletion of $\sim 2 \mathrm{mM}$ of GlcNAc during this period matched the accumulation of pyruvate, lactate, and acetate, each at $\sim 2 \mathrm{mM}$. These data can be rationalized by the simple metabolic picture shown in Extended Data Fig. 5, in which the GlcNAc influx is largely turned into equal molar excretion of acetate, pyruvate and lactate. In this scenario, pyruvate/lactate excretion results from blockage at the end of glycolysis (where pyruvate is converted to Ac-coA), due to a high internal pool of AccoA which is connected by reversible reactions to the high acetic acid concentration in the medium ${ }^{41,42}$.

The build-up of acetate in the cytoplasm also suggested a mechanism for the excretion of other internal metabolites required for osmolarity balance, with glutamate being the easiest to detect due to its high cytoplasmic concentration ${ }^{43}$. Indeed, untargeted metabolomic analysis on the spent medium of $1 \mathrm{~A} 01$ monoculture showed the increase of numerous internal metabolites (Extended Data Fig. 6). To see whether such metabolites may also be cross-fed in the coculture, we analyzed the spent media collected from dense sampling using untargeted metabolomics ${ }^{44,45}$, and found many internal metabolites with similar dynamics as those in Fig. 2e (purple curves, Fig. 2f). Altogether, these data show that internal metabolites were extensively excreted by $1 \mathrm{~A} 01$ during acetate stress and were cross-fed to 3B05.

\section{Growth recovery after dilution into fresh medium}

Differential lag can play important roles in maintaining coexistence ${ }^{46}$. Curiously, the lag of 1 A01 following dilution in the stable cycle occurred even though it experienced no acetate stress since the second half of the previous cycle (Fig. 2a,b). We hypothesized that the lag of 1A01 resulted from the brief exposure to acetate stress during the previous cycle. To test this, we grew 1A01 monoculture in GlcNAc with the weak buffer and allowed it to self-acidify the medium (Fig. 4a). When the culture reached pH 5 (dashed vertical line), we washed and resuspended the cells in fresh GlcNAc medium and followed the OD of the fresh culture (Fig. 4b). The culture did not grow for at least 6 hours (open black squares, Fig. 4b). In contrast, cells taken from a little earlier (at pH 5.3, indicated by the dotted vertical line in Fig. 4a), lagged but started growing again after $4 \mathrm{~h}$ (filled black squares, Fig. 4b), while cells taken from exponential growth at normal $\mathrm{pH}$ grew immediately (filled grey squares, Fig. 4b), and cells taken from stationary phase in strong buffer (normal pH) grew after a short lag (open light grey squares, Fig. 4b). Thus, long lag in the growth recovery of 1A01 can indeed result from a 1-2 h exposure to low $\mathrm{pH}$ as it occurred in the stable cycle. Analysis of the spent media during the lag period revealed that GlcNAc was taken up despite the lack of growth by 1A01, while acetate, lactate, and pyruvate were excreted (Fig. 4c), at sufficient amount to account for the growth of 3B05 observed in the first 6 hours of the stable cycle (Fig. 2a).

The lag exhibited by $1 \mathrm{~A} 01$ cells taken at $\mathrm{pH} 5$ was completely relieved if GlcNAc was supplemented by fresh marine broth (Fig. 4d, filled magenta squares), indicating that acid-shocked 1A01 cells were not intrinsically limited from re-growth. Rather some metabolites were lacking in the transition period, presumably resulting from the excretion of internal metabolites while being in acetate (Fig. 2e-f, 3f), as discussed in Extended Data Fig. 5. Since pyruvate and acetate were excreted during the initial period of regrowth (Fig. 4c), we reasoned that cells lagged due to shortage of TCA intermediates or metabolites derived from these intermediates (Fig. 4e). We thus quantified the abundances of related amino acids (indicated by purple boxes) and found indeed that a number of them were depleted upon exposure to acetate (Fig. 4f), with aspartate being the most depleted. Strikingly, supplementing the recovery medium with aspartate (but not others) allowed much faster recovery (open symbols, Fig. 4d). Thus, the lag of 1A01 is an intrinsic physiological 
problem suffered by acetate-shocked cells lasting well beyond the duration of the stress. It indicates a metabolic memory of the stressed cells that takes time to overcome, requiring the consumption of nutrients (GlcNAc in this case), presumably to replenish the pools of key excreted internal metabolites.

\section{Approach to the stable cycle and dependence on the initial inoculation}

Key ingredients maintaining the stable cycle from the above experiments were integrated into a mathematical model of cross-feeding constrained by measured kinetic parameters (Fig. 5a, Supp Note 3). The model was able to capture the stable cycle dynamics quantitatively, including the timing and magnitude of the major metabolites around the acetate peak and the densities of the two species (Fig. 5b, compare to Fig. 2a, 2b; see Extended Data Fig. 7a for a detailed description). Moreover, the model also reproduced the strain abundances and GlcNAc/acetate concentrations at the end of each cycle as the coculture approached the stable cycle (Fig. 5c, compare to Fig. 1d, 1f). Additionally, the model depicted a complex sequence during the first three cycles of adaptation (Fig. 5d), featuring the massive death of 1A01 in the first cycle, followed by a prolonged build-up of 1A01 cells in the second cycle, before locking into the stable cycle (see description in Extended Data Fig. 7c-d).

Exploration of the model suggested shorter approaches to the stable cycle if the initial inoculant was tilted more towards 3B05, with initial density ratio of 3B05:1A01 = 3:1 putting the system immediately in the stable cycle (Extended Data Fig. 8a). Repeating the 24-h growth-dilution experiment of Fig. 1a for this initial ratio resulted in the same stable cycle dynamics including the acetate peak and transient accumulation of internal metabolites (Extended Data Fig. 8b), with comparable viable cell densities (Extended Data Fig. 8c). Moreover, this stable cycle was achieved immediately after the first cycle as predicted (Extended Data Fig. 8d-e, 7b). These results establish experimentally that features of the stable cycle are properties of the community, independent of the initial condition and transient dynamics such as massive cell death.

\section{Discussion}

Detailed quantitative analysis revealed complex metabolic interplays underlying a simple coculture of two bacterial species. Coexistence found in growth-dilution experiments occurred dynamically as a limit cycle, involving a transition from preferential growth of $1 \mathrm{~A} 01$ in unstressed condition to its subsequent acidification and internal metabolite excretion, followed by stress relief by 3B05. As acidification due to acetate and other fermentation products occurs widely in microbial communities ${ }^{47-53}$ and further, as the excretion of internal metabolites is an obligatory physiological response by bacteria under weak acid stress ${ }^{41,42}$, we expect the basic features of our model system to apply generally across microbial ecosystems, including those being widely used for ecological studies ${ }^{4,54}$.

In this context, we note that while extensive cross-feeding during steady-state growth has been hypothesized as a general mechanism to support the coexistence of many species ${ }^{4-6}$, physiological rationales are lacking for organisms to excrete metabolites altruistically to support the growth of others in conditions where each can grow alone: Although substantial metabolic overflow by fast-growing microbes is well known, the diversity of such overflow metabolites is quite limited ${ }^{55,56}$. Excretion of diverse metabolites is reported also during slow exponential growth ${ }^{57}$, but the amount is limited and is insufficient to support substantial biomass accumulation by other species. Extensive excretion of internal metabolites does occur under various nutrient limiting conditions, e.g., carbon catabolites under nitrogen limitation $38,58,59$; but this would not benefit other organisms experiencing the same limitation. Thus, studies of mutualistic cross-feeding have mostly involved systems in which individual species cannot grow alone, such as anaerobic digesters constrained by extremely low energy influx ${ }^{11,12}$, and synthetic or evolved auxotrophs ${ }^{17-25}$. 
Here, we find extensive cross-feeding of internal metabolites to occur transiently during stress-imposed growth arrest, with the only apparent cost to the excreting cells being a moderate lag period after the stress is relieved. Thus the transient excretion of internal metabolites, by stressed cells that cannot grow anyway, offers other species an opportunity to grow and (hopefully) remove the source of stress. Importantly, coexistence which occurred in the 24-h growth-dilution cycles featuring extended growth arrest, failed to establish in the same system under 6-h cycles that provided a chemostat-like, stress-free growth condition. The results therefore present a rare, mechanistically explicit validation of the Stress Gradient Hypothesis, which predicts that positive interactions are facilitated during stress rather than during times of plenty ${ }^{60-62}$. 284 Thus stress-induced cross-feeding offers a general, dynamical mechanism for a community to overcome 285 stress while simultaneously opening up many metabolic niches ${ }^{5,6}$, with the consequence of promoting coexistence of a large number of species even in environments supplied with only one or a few nominal resources $^{4,26}$. 


\section{References}

1. Morris, B. E. L., Henneberger, R., Huber, H. \& Moissl-Eichinger, C. Microbial syntrophy: Interaction for the common good. FEMS Microbiology Reviews vol. 37 384-406 (2013).

2. Wintermute, E. H. \& Silver, P. A. Dynamics in the mixed microbial concourse. Genes and Development vol. 24 2603-2614 (2010).

3. Smith, N. W., Shorten, P. R., Altermann, E., Roy, N. C. \& McNabb, W. C. The Classification and Evolution of Bacterial Cross-Feeding. Front. Ecol. Evol. 7, 153 (2019).

4. Goldford, J. E. et al. Emergent simplicity in microbial community assembly. Science (80-. ). 361, 469-474 (2018).

5. Pacheco, A. R., Moel, M. \& Segrè, D. Costless metabolic secretions as drivers of interspecies interactions in microbial ecosystems. Nat. Commun. 10, 1-12 (2019).

6. Marsland, R., Cui, W. \& Mehta, P. A minimal model for microbial biodiversity can reproduce experimentally observed ecological patterns. Sci. Rep. 10, 1-17 (2020).

7. Hardin, G. The competitive exclusion principle. Science (80-. ). 131, 1292-1297 (1960).

8. Hutchinson, G. E. The Paradox of the Plankton. Am. Nat. 95, 137-145.

9. MacArthur, R. Species packing and competitive equilibrium for many species. Theor. Popul. Biol. 1, $1-11$ (1970).

10. Winogradsky, S. Recherches sur les organismes de la nitrification. Ann. Inst. Pasteur 4, 213-231 (1890).

11. Thauer, R. K., Jungermann, K. \& Decker, K. Energy conservation in chemotrophic anaerobic bacteria. Microbiol. Mol. Biol. Rev. 41, (1977).

12. Schink, B. Energetics of Syntrophic Cooperation in Methanogenic Degradation. MICROBIOLOGY AND MOLECULAR BIOLOGY REVIEWS vol. 61 (1997).

13. Costa, E., Pérez, J. \& Kreft, J. U. Why is metabolic labour divided in nitrification? Trends Microbiol. 14, 213-219 (2006).

14. Udvardi, M. \& Poole, P. S. Transport and Metabolism in Legume-Rhizobia Symbioses. Annu. Rev. Plant Biol. 64, 781-805 (2013).

15. Croft, M. T., Lawrence, A. D., Raux-Deery, E., Warren, M. J. \& Smith, A. G. Algae acquire vitamin B12 through a symbiotic relationship with bacteria. Nature 438, 90-93 (2005).

16. Amin, S. A. et al. Photolysis of iron-siderophore chelates promotes bacterial-algal mutualism. Proc. Natl. Acad. Sci. U. S. A. 106, 17071-17076 (2009).

17. Shou, W., Ram, S. \& Vilar, J. M. G. Synthetic cooperation in engineered yeast populations. Proc. Natl. Acad. Sci. U. S. A. 104, 1877-1882 (2007).

18. Harcombe, W. Novel cooperation experimentally evolved between species. Evolution (N. Y). 64, 2166-2172 (2010).

19. Mee, M. T., Collins, J. J., Church, G. M. \& Wang, H. H. Syntrophic exchange in synthetic microbial communities. Proc. Natl. Acad. Sci. U. S. A. 111, E2149-E2156 (2014).

20. Hoek, T. A. et al. Resource Availability Modulates the Cooperative and Competitive Nature of a Microbial Cross-Feeding Mutualism. PLOS Biol. 14, e1002540 (2016).

21. Dolinšek, J., Goldschmidt, F. \& Johnson, D. R. Synthetic microbial ecology and the dynamic interplay between microbial genotypes. FEMS Microbiology Reviews vol. 40 961-979 (2016).

22. Lilja, E. E. \& Johnson, D. R. Segregating metabolic processes into different microbial cells accelerates the consumption of inhibitory substrates. ISME J. 10, 1568-1578 (2016).

23. Harcombe, W. R., Chacón, J. M., Adamowicz, E. M., Chubiz, L. M. \& Marx, C. J. Evolution of bidirectional costly mutualism from byproduct consumption. Proc. Natl. Acad. Sci. U. S. A. 115, 12000-12004 (2018). 
24. Co, A. D., van Vliet, S., Kiviet, D. J., Schlegel, S. \& Ackermann, M. Short-range interactions govern the dynamics and functions of microbial communities. Nat. Ecol. Evol. 4, 366-375 (2020).

25. Green, R. et al. Metabolic excretion associated with nutrient-growth dysregulation promotes the rapid evolution of an overt metabolic defect. PLoS Biol. 18, e3000757 (2020).

26. Dal Bello, M., Lee, H., Goyal, A. \& Gore, J. A simple linear relationship between resource availability and microbial 1 community diversity. bioRxiv 2020.09.12.294660 (2020) doi:10.1101/2020.09.12.294660.

27. Thompson, L. R. et al. A communal catalogue reveals Earth's multiscale microbial diversity. Nature 551, 457-463 (2017).

28. Sunagawa, S. et al. Structure and function of the global ocean microbiome. Science (80-. ). 348 , (2015).

29. Huttenhower, C. et al. Structure, function and diversity of the healthy human microbiome. Nature 486, 207-214 (2012).

30. Datta, M. S., Sliwerska, E., Gore, J., Polz, M. F. \& Cordero, O. X. Microbial interactions lead to rapid micro-scale successions on model marine particles. Nat. Commun. 7, 1-7 (2016).

31. Hofmann, G. E. et al. High-Frequency Dynamics of Ocean pH: A Multi-Ecosystem Comparison. PLoS One 6, e28983 (2011).

32. Cermak, N., Datta, M. Sen \& Conwill, A. Rapid, Inexpensive Measurement of Synthetic Bacterial Community Composition by Sanger Sequencing of Amplicon Mixtures. iScience 23, (2020).

33. Bassler, B. L., Yu, C., Lee, Y. C. \& Roseman, S. Chitin utilization by marine bacteria: Degradation and catabolism of chitin oligosaccharides by Vibrio furnissii. J. Biol. Chem. 266, 24276-24286 (1991).

34. Fadda, H. M. et al. Drug solubility in luminal fluids from different regions of the small and large intestine of humans. Mol. Pharm. 7, 1527-1532 (2010).

35. Fierer, N. \& Jackson, R. B. The diversity and biogeography of soil bacterial communities. Proc. Natl. Acad. Sci. U. S. A. 103, 626-631 (2006).

36. Butler, J. N. Carbon dioxide equilibria and their applications. (Addison-Wesley, 1982).

37. Estrela, S., Trisos, C. H. \& Brown, S. P. From Metabolism to Ecology: Cross-Feeding Interactions Shape the Balance between Polymicrobial Conflict and Mutualism. Am. Nat. 180, 566-576 (2012).

38. McCully, A. L., LaSarre, B. \& McKinlay, J. B. Growth-independent cross-feeding modifies boundaries for coexistence in a bacterial mutualism. Environ. Microbiol. 19, 3538-3550 (2017).

39. Ratzke, C. \& Gore, J. Modifying and reacting to the environmental $\mathrm{pH}$ can drive bacterial interactions. PLOS Biol. 16, e2004248 (2018).

40. Ratzke, C., Denk, J. \& Gore, J. Ecological suicide in microbes. Nat. Ecol. Evol. 2, 867-872 (2018).

41. Russell, J. B. \& Diez-Gonzalez, F. The effects of fermentation acids on bacterial growth. $A d v$. Microb. Physiol. 39, 228-234 (1998).

42. Roe, A. J., McLaggan, D., Davidson, I., O’Byrne, C. \& Booth, I. R. Perturbation of anion balance during inhibition of growth of Escherichia coli by weak acids. J. Bacteriol. 180, 767-772 (1998).

43. Bennett, B. D. et al. Absolute metabolite concentrations and implied enzyme active site occupancy in Escherichia coli. Nat. Chem. Biol. 5, 593-599 (2009).

44. Fuhrer, T., Heer, D., Begemann, B. \& Zamboni, N. High-throughput, accurate mass metabolome profiling of cellular extracts by flow injection-time-of-flight mass spectrometry. Anal. Chem. 83, 7074-7080 (2011).

45. Pontrelli, S. \& Sauer, U. Salt-Tolerant Metabolomics for Exometabolomic Measurements of Marine Bacterial Isolates. Anal. Chem. (2021) doi:10.1021/acs.analchem.0c04795.

46. Manhart, M. \& Shakhnovich, E. I. Growth tradeoffs produce complex microbial communities on a 
single limiting resource. Nat. Commun. 9, 1-9 (2018).

47. Fukuzaki, S., Nishio, N., Shobayashi, M. \& Nagai, S. Inhibition of the Fermentation of Propionate to Methane by Hydrogen, Acetate, and Propionate. Appl. Environ. Microbiol. 56, (1990).

48. Eiteman, M. A. \& Altman, E. Overcoming acetate in Escherichia coli recombinant protein fermentations. Trends Biotechnol. 24, 530-536 (2006).

49. Warnecke, T. \& Gill, R. T. Organic acid toxicity, tolerance, and production in Escherichia coli biorefining applications. Microbial Cell Factories vol. 425 (2005).

50. Macfarlane, S. \& Macfarlane, G. T. Regulation of short-chain fatty acid production. Proc. Nutr. Soc. 62, 67-72 (2003).

51. Blasche, S. et al. Metabolic cooperation and spatiotemporal niche partitioning in a kefir microbial community. Nat. Microbiol. 6, 196-208 (2021).

52. Drzyzga, O. \& Gottschal, J. C. Tetrachloroethene dehalorespiration and growth of Desulfitobacterium frappieri TCE1 in strict dependence on the activity of Desulfovibrio fructosivorans. Appl. Environ. Microbiol. 68, 642-649 (2002).

53. Senior, E., Bull, A. T. \& Slater, J. H. Enzyme evolution in a microbial community growing on the herbicide Dalapon. Nature 263, 476-479 (1976).

54. Estrela, S. et al. Metabolic rules of microbial community assembly. bioRxiv 2020.03.09.984278 (2020) doi:10.1101/2020.03.09.984278.

55. Basan, M. et al. Overflow metabolism in Escherichia coli results from efficient proteome allocation. Nature 528, 99-104 (2015).

56. van Dijken, J. P., Weusthuis, R. A. \& Pronk, J. T. Kinetics of growth and sugar consumption in yeasts. Antonie Van Leeuwenhoek 63, 343-352 (1993).

57. Paczia, N. et al. Extensive exometabolome analysis reveals extended overflow metabolism in various microorganisms. Microb. Cell Fact. 11, 122 (2012).

58. Folsom, J. P. \& Carlson, R. P. Physiological, biomass elemental composition and proteomic analyses of Escherichia coli ammoniumlimited chemostat growth, and comparison with iron- and glucoselimited chemostat growth. Microbiol. (United Kingdom) 161, 1659-1670 (2015).

59. Kochanowski, K. et al. Global coordination of metabolic pathways in Escherichia coli by active and passive regulation. Mol. Syst. Biol. 17, e10064 (2021).

60. Hammarlund, S. P. \& Harcombe, W. R. Refining the stress gradient hypothesis in a microbial community. Proceedings of the National Academy of Sciences of the United States of America vol. 116 15760-15762 (2019).

61. Piccardi, P., Vessman, B. \& Mitri, S. Toxicity drives facilitation between 4 bacterial species. Proc. Natl. Acad. Sci. U. S. A. 116, 15979-15984 (2019).

62. Bertness, M. D. \& Callaway, R. Positive interactions in communities. Trends in Ecology and Evolution vol. 9 191-193 (1994).

63. Keseler, I. M. et al. EcoCyc: A comprehensive database of Escherichia coli biology. Nucleic Acids Res. 39, 583-590 (2011).

Acknowledgments: We are grateful to helpful discussions with numerous colleagues during the course of this work, including Jeff Gore, Shaul Pollak, Wenying Shou, Alfred Spormann, and members of the Hwa lab, particularly Hiro Okano for technical assistance. This work is supported by the Simons Foundation through the Principles of Microbial Ecosystems (PriME) collaboration (Grant no. 542387 to TH, 542395 to OXC, 608247 to SP). KA acknowledges a Simons PriME fellowship (Grant no. 647858). JD acknowledges the hospitality of UCSD during her sabbatical visit, and the support of the NSF through grants DMR1703231 and MCB-2029480. 

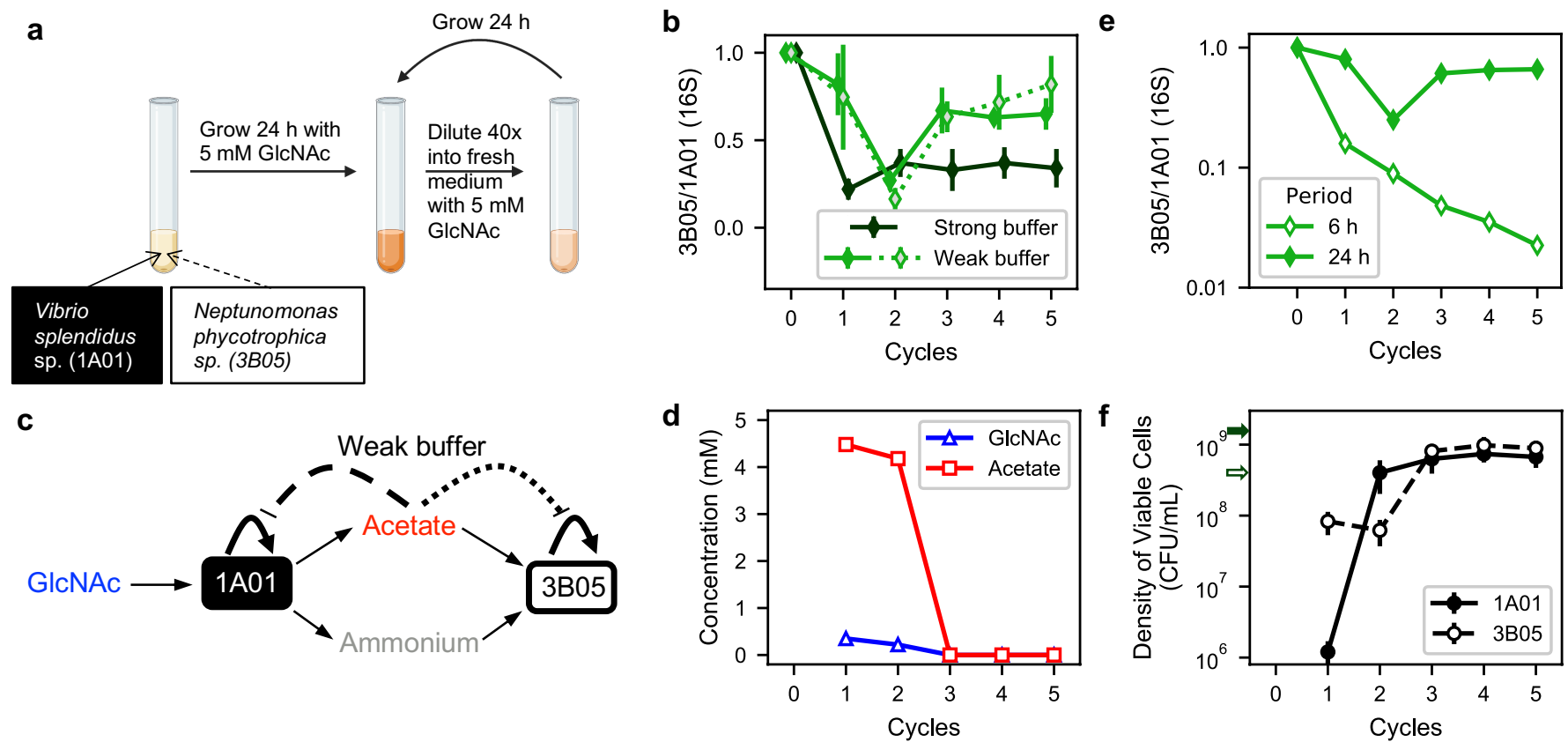

Figure 1. Coexistence of $1 \mathrm{A01}$ and $3 \mathrm{B05}$ in growth-dilution cycles. Vibrio splendidus sp. 1A01 and Neptunomonas phycotrophica sp. 3B05 in growth-dilution cycles with $5 \mathrm{mM}$ GlcNAc as the sole carbon and nitrogen source and 40x dilution after each cycle. (b) Ratio of 3B05 to 1A01 based on 16S reads at the end of each cycle, starting from 1:1 mixture of exponentially growing cells each at $\mathrm{OD}_{600}=0.01$. Results from experiments done in strong buffer (40 mM HEPES) are shown in black, and those from experiments done in weak buffer $\left(2 \mathrm{mM} \mathrm{NaHCO}_{3}\right)$ are shown in green. The weak buffer data with open symbols was from a coculture initiated using clones from culture collected at the end of a previous round of 5 growth-dilution cycles in weak buffer. (c) Schematic of acetate cross-feeding based on single-strain characteristics derived from Extended Data Fig. 1. In the weak buffer, acetate excretion provides a canonical scenario of syntrophy if acidification inhibits 1A01 more than 3B05; see dashed line and also Extended Data Fig. 2a. However, acidification actually inhibits 3B05 more strongly (dotted line and Extended Data Fig. 2b), indicating that the canonical scenario does not hold here. (d) Concentrations of GlcNAc (blue triangles) and acetate (red squares) in the medium measured at the end of each 24-h growth period for the coculture grown in weak buffer. In the strongly-buffered case, no GlcNAc or Acetate is left in the medium from Cycle 1 onwards. (e) Ratio of 3B05 to $1 \mathrm{~A} 01$ 16S reads collected at the end of each cycle of a 6-h growth/dilution experiments in the weak buffer (open diamonds). Results for the 24-h cycle (filled green diamonds) are reproduced from panel b for comparison. (f) Viable cell count of 1A01 (filled circles) and 3B05 (open circles) at the end of each 24-hr growth period for the coculture grown in weak buffer. Error bars indicate variation between replicate experiments. Results for the same quantities for a coculture grown in strong buffer are constant over several growth-dilution cycles and are shown as the filled and open arrows on the left axis, $1.6 \times 10^{9} \mathrm{CFU} / \mathrm{ml}$ for $1 \mathrm{~A} 01$ and $4 \times 10^{8} \mathrm{CFU} / \mathrm{ml}$ for $3 \mathrm{~B} 05$, respectively. Note that the ratio of viable count $(3 \mathrm{~B} 05: 1 \mathrm{~A} 01)$ was higher in weak buffer compared to that in strong buffer. 


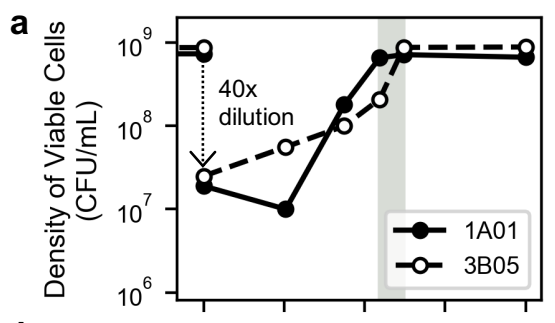

b

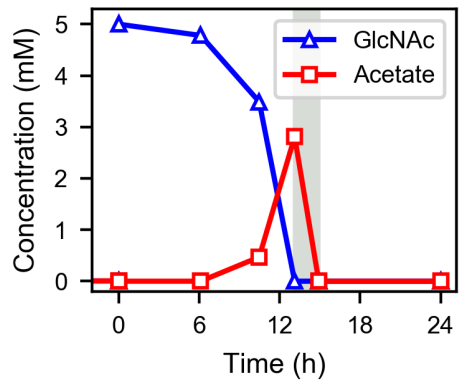

d
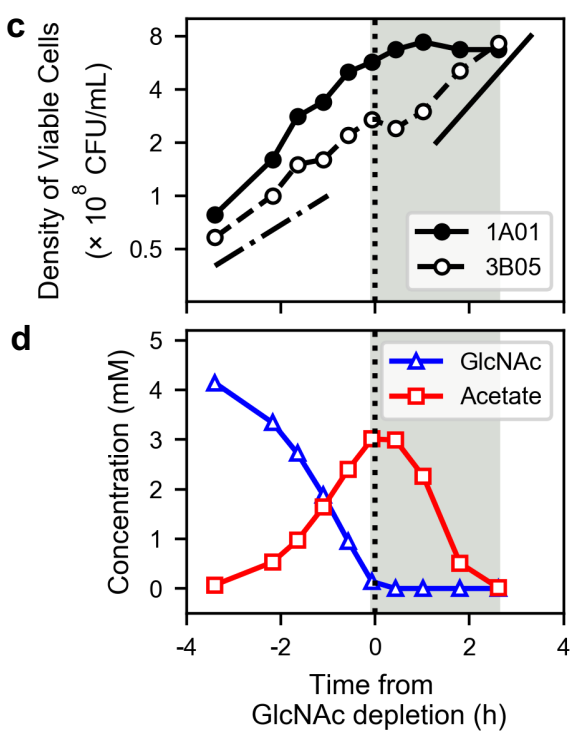
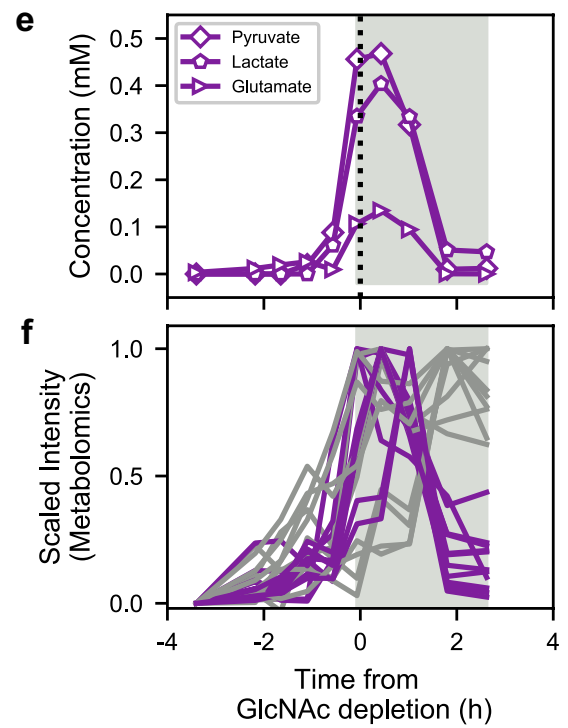

451

452

453

454

455

456

457

458

459

460

461

462

463

464

465

466

Figure 2. Cross-feeding in the stable cycle of the coculture in weak buffer. Measurements of various quantities of the coculture throughout the fifth 24-h growth/dilution cycle. (a) The viable counts of $1 \mathrm{~A} 01$ and 3B05 cells. (b) The concentrations of GlcNAc and Acetate in the medium. The light grey regions in (a) and (b) indicate the period when 3B05 continued to grow after GlcNAc depletion. In (c)-(f), the duration around the 'acetate peak' were densely sampled using a protocol that mimicked the stable cycle; see Methods. The dotted line at time " 0 " indicates the time of GlcNAc depletion, around $12 \mathrm{~h}$ into the cycle (panel b). Grey shaded regions are the same as those in panels (a) and (b). Same symbols are used in (c) and (d) as in (a) and (b). In (c) the dashed-dotted line indicates exponential growth of $3 \mathrm{~B} 05$ at rate $\sim 0.35 / \mathrm{hr}$ before the acetate peak; the solid line indicates a growth rate $\sim 0.55 / \mathrm{hr}$ after the acetate peak. (e) Concentrations of pyruvate, lactate, and glutamate in the medium as measured by HPLC. (f) Scaled intensities of metabolites in the medium as measured by untargeted metabolomics; see Methods. Metabolites consumed (defined as those with the scaled intensity of the last time point $<0.5$ ) are plotted in purple. Other detected metabolites are plotted in grey. Identities of the metabolites are shown in Extended Data Table 2. 

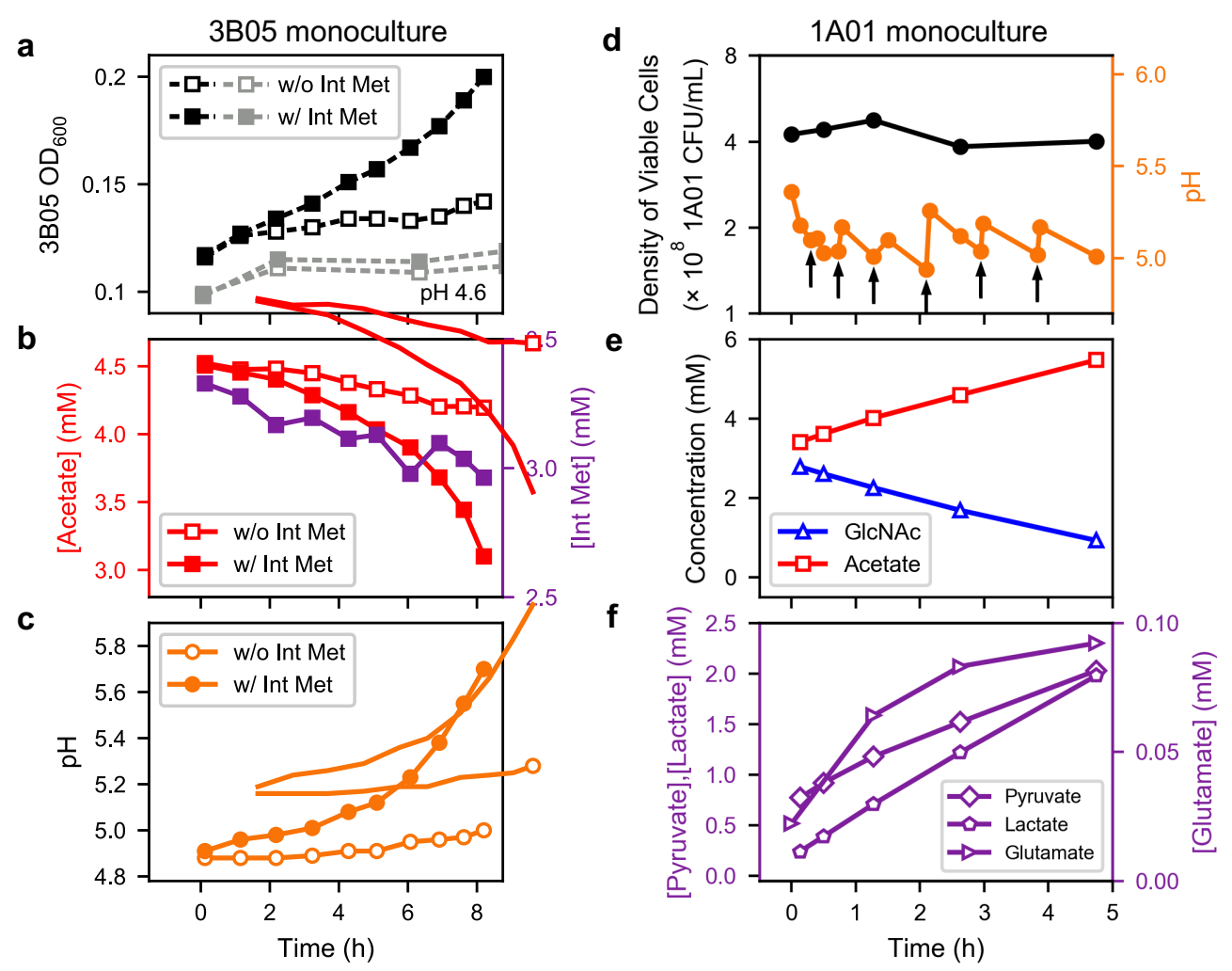

Figure 3. Metabolism under acetate stress. (a)-(c) 3B05 was precultured alone in strongly buffered acetate medium. Then the culture was washed and transferred to weakly buffered medium $\left(2 \mathrm{mM} \mathrm{NaHCO}_{3}\right)$ with 4.5 $\mathrm{mM}$ sodium acetate, with (filled symbols) or without (open symbols) the addition of pyruvate, lactate and glutamate ("Int Met") (a) OD, with the grey symbols showing results from the same experiment starting at a lower $\mathrm{pH}$ (by the addition of $\mathrm{HCl}$ ), (b) acetate (left axis) and the sum of pyruvate, lactate and glutamate concentrations (right axis) in the medium, and (c) $\mathrm{pH}$ of the medium. (d)-(f) Exponentially growing 1A01 monoculture was initiated at $\mathrm{OD}_{600}=0.2$ and grew in steady-state in GlcNAc medium with the weak buffer $\left(2 \mathrm{mM} \mathrm{NaHCO}_{3}\right)$ until acetate excretion dropped the $\mathrm{pH}$ to $\sim 5$ where OD reached 0.45 , corresponding to viable cell density of $\sim 4 \times 10^{8} \mathrm{CFU} / \mathrm{mL}$. The $\mathrm{pH}$ was then maintained in a narrow $\mathrm{pH}$ range by titrating with $0.1 \mathrm{M} \mathrm{NaHCO}_{3}$. (d) Viable cell count (black circles) and $\mathrm{pH}$ (orange circles); the arrows indicate times at which $\mathrm{NaHCO}_{3}$ was added. (e) Concentrations of GlcNAc (blue triangles) and acetate (red squares) in the medium. (f) Concentrations of Pyruvate, Lactate, and Glutamate in the medium. 

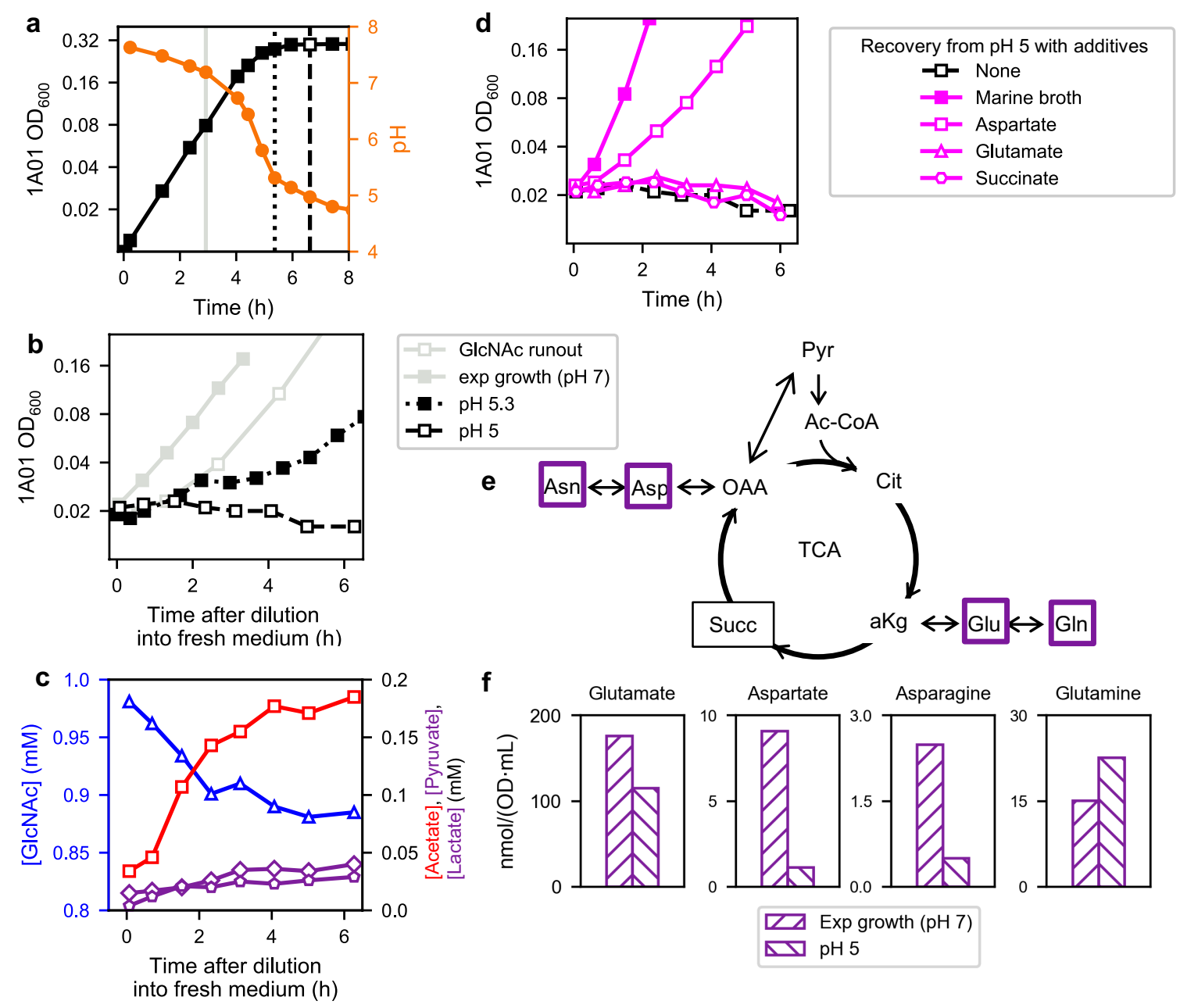

483

484

485

486

487

488

489

490

491

492

493

494

495

496

497

498

499

500

Figure 4. Recovery of $1 \mathrm{A01}$ growth after experiencing acetate stress. (a) Growth of 1A01 monoculture in $5 \mathrm{mM}$ GlcNAc with the weak $2 \mathrm{mM}$ bicarbonate buffer. Samples of culture were taken at the time indicated by the dotted and dashed lines, capturing exposure to different $\mathrm{pH}$ levels (5.3 and 5.0), as well as at normal $\mathrm{pH}$ (solid grey line). (b) The cells taken from these different time points in (a) were washed and resuspended in fresh GlcNAc medium with $2 \mathrm{mM}$ bicarbonate, $\mathrm{pH} \sim 7.5$. The open grey symbols indicate $1 \mathrm{~A} 01$ cells that spent $\sim 12 \mathrm{~h}$ in strongly buffered medium following GlcNAc runout and experienced no acid stress before resuspension in fresh strongly buffered GlcNAc medium. (c) Concentrations of GlcNAc (open triangles, left axis), Acetate (red squares), Pyruvate (purple diamonds), and Lactate (purple pentagons) in the medium during the lag period for cells taken from $\mathrm{pH}$ 5. (d) The experiment described in (b) was repeated using cells taken from $\mathrm{pH} 5$, except that the fresh GlcNAc medium was supplemented by $100 \%$ marine broth (solid magenta squares), $5 \mathrm{mM}$ aspartate (open magenta squares), $5 \mathrm{mM}$ glutamate (open magenta triangles), and 5 $\mathrm{mM}$ succinate (open magenta hexagon). Open black squares are the same as those shown in (b) for reference. (e) Schematic showing the TCA cycle and amino acids (boxed) immediately derived from TCA intermediates. (f) Cellular content of the amino acids shown in (e) during exponentially growing (right hash) and growth arrested at $\mathrm{pH} 5$ (left hash) cells. 
a

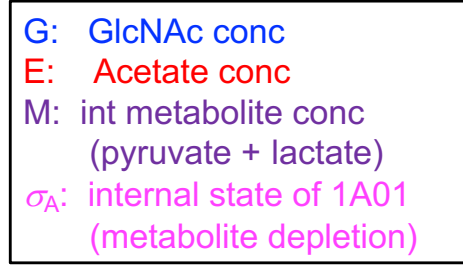

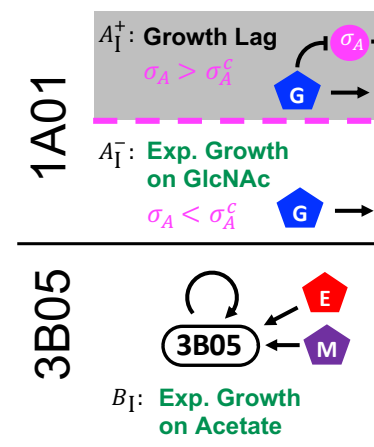
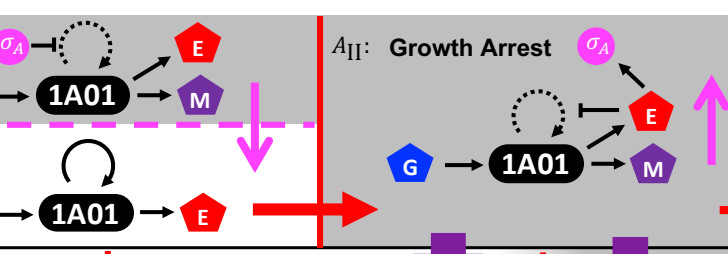

$A_{\text {III }}$ Death

(A01)
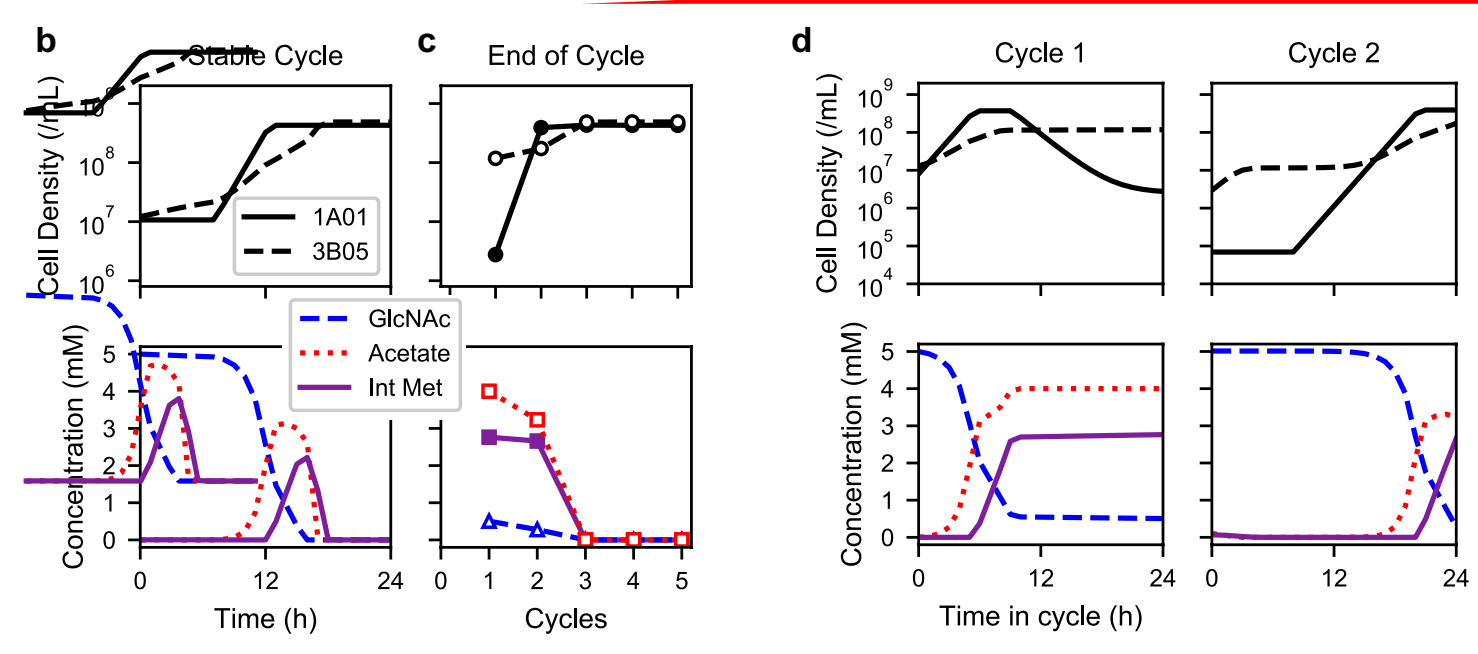

Figure 5. Model of stress-induced cross-feeding and the resulting population dynamics in 24-h growthdilution cycles. (a) We describe the cross-feeding dynamics by a consumer-resource model outside of the realm of steady state that is typically considered. The model involves the densities of $1 \mathrm{~A} 01$ and 3B05 cells and the concentrations of GlcNAc $(\mathrm{G})$, acetate $(\mathrm{E})$, and internal metabolite $(\mathrm{M}$, to be interpreted as the sum of pyruvate and lactate concentrations in the medium). We also introduce an additional variable $\sigma_{A}$ to describe the internal state of $1 \mathrm{~A} 01$ due to the depletion of other internal metabolites such as aspartate and glutamate (Fig. 4). The key feature of our model is that the growth/death rate of the two species and the rates of uptake/excretion of the metabolites $\mathrm{G}, \mathrm{E}$, and $\mathrm{M}$ by the two species are dependent on the degree of acetate stress (E) and 1A01's internal state $\left(\sigma_{A}\right)$. As illustrated in the schematic, we approximate these dependences of the rate functions by switching between several distinct forms depending on the values of $\sigma_{A}$ and $\mathrm{E}$ corresponding to each of the four regimes for $1 \mathrm{~A} 01\left(A_{\mathrm{I}}^{+}, A_{\mathrm{I}}^{-}, A_{\mathrm{II}}, A_{\mathrm{III}}\right)$ and the value of $\mathrm{E}$ which corresponding to each of the three regimes for $3 \mathrm{~B} 05\left(B_{\mathrm{I}}, B_{\mathrm{II}}, B_{\mathrm{III}}\right)$. These regimes are separated by the vertical red lines and horizontal dashed magenta line; see Supp Note 3 for a detailed description. In the schematic, black arrows with solid and dashed lines indicate effective and ineffective interactions in each regime. Red arrows indicate the change in the acetate concentration $\mathrm{E}$, and the magenta arrows indicate the change of $\sigma_{A}$. Thick purple arrows indicate the crucial cross-feeding of internal metabolites allowing 3B05 to grow during acetate stress (and hence reduce the acetate concentration in the medium). Panels (b)-(d) show numerical results of the model for the density of live 1A01 and 3B05 cells (top) and the concentration of GlcNAc, acetate, and internal metabolites (bottom) for the 24-h growth-dilution cycles with 1:1 initial species ratio. (b) Numerical results of the model during the stable cycle, which leads to two coordinated paths, $A_{\mathrm{I}}^{+} \rightarrow A_{\mathrm{I}}^{-} \rightarrow A_{\mathrm{II}} \rightarrow A_{\mathrm{I}}^{+}$for $1 \mathrm{~A} 01$ and $B_{\mathrm{I}} \rightarrow B_{\mathrm{II}} \rightarrow B_{\mathrm{I}}$ for 3B05 over time. (c) Numerical results of the model at the end of each 24-hr cycle. 
522 (d) Numerical results of the model during the first 3 cycles. In the first cycle, $1 \mathrm{~A} 01$ took the path $A_{\mathrm{I}}^{-} \rightarrow A_{\mathrm{II}} \rightarrow$ $523 A_{\text {III }}$ into the death phase, while 3B05 took the path $B_{\mathrm{I}} \rightarrow B_{\mathrm{II}} \rightarrow B_{\mathrm{III}}$ into growth arrest. Paths that go into $A_{\mathrm{III}}$ 524 and $B_{\text {III }}$ cannot recover in the same cycle but can recover in subsequent cycles as acetate is diluted and 525 GlcNAc is provided in a new cycle. See Extended Data Fig. 7 for a detailed description of the dynamics. 


\section{Extended Data Table and Figures}

\begin{tabular}{|c|c|c|c|c|c|c|c|}
\hline Strain & $\begin{array}{c}\text { Carbon \& } \\
\text { Nitrogen } \\
\text { source }\end{array}$ & $\begin{array}{l}\text { Growth } \\
\text { rate } \\
\left(\mathrm{h}^{-1}\right)\end{array}$ & $\begin{array}{l}\text { Inverse biomass } \\
\text { yield on carbon } \\
\text { substrate } \\
(\mathrm{mM} / \mathrm{OD})\end{array}$ & $\begin{array}{l}\text { Acetate } \\
\text { excretion } \\
(\mathrm{mM} / \mathrm{OD})\end{array}$ & $\begin{array}{l}\text { Ammonium } \\
\text { excretion } \\
\text { (mM/OD) }\end{array}$ & $\begin{array}{c}\text { Inverse } \\
\text { biomass } \\
\text { yield on } \\
\text { ammonium } \\
\text { (mM/OD) }\end{array}$ & $\mathrm{CFU} / \mathrm{OD} / \mathrm{mL}$ \\
\hline \multirow{2}{*}{$\begin{array}{l}\text { V. splendidus } \\
\text { sp. 1A01 }\end{array}$} & GlcNAc & $0.82 \pm 0.05$ & $6.1 \pm 0.2$ & $9.3 \pm 0.3$ & $1.4 \pm 0.2$ & - & \multirow[t]{2}{*}{$8.0 \pm 0.2 \mathrm{E} 8$} \\
\hline & $\begin{array}{l}\text { Glucose, } \\
\text { Ammonium }\end{array}$ & $0.83 \pm 0.04$ & $5.7 \pm 0.3$ & $2.3 \pm 0.2$ & - & $4.1 \pm 0.2$ & \\
\hline $\begin{array}{l}\text { N. phycotrophica } \\
\text { sp. 3B05 }\end{array}$ & $\begin{array}{c}\text { Acetate, } \\
\text { Ammonium } \\
\text { Acetate, } \\
\text { Ammonium, } \\
\text { Lactate, } \\
\text { Pyruvate, } \\
\text { Glutamate } \\
\end{array}$ & $0.45 \pm 0.03$ & $32 \pm 2.4$ & - & - & $4.1 \pm 0.2$ & $1.3 \pm 0.2 \mathrm{E} 9$ \\
\hline \multirow{2}{*}{$\begin{array}{l}\text { E. coli } \mathrm{K}-12 \\
\text { NCM3722 }\end{array}$} & GlcNAc & $0.57 \pm 0.03$ & $5.1 \pm 0.4$ & $10.5 \pm 0.4$ & $2.1 \pm 0.3$ & & \\
\hline & $\begin{array}{l}\text { Glucose, } \\
\text { Ammonium }\end{array}$ & $0.59 \pm 0.04$ & $5.6 \pm 0.2$ & $3.9 \pm 0.1$ & - & & \\
\hline
\end{tabular}

Extended Data Table 1. Monoculture steady-state physiological parameters for $V$. splendidus sp. 1A01, $N$. phycotrophica sp. 3B05, and E. coli NCM3722. 1A01 and 3B05 were grown in the strongly buffered HEPES medium described in Materials and Methods; all parameters were the same in the weakly buffered medium with $2 \mathrm{mM}$ sodium bicarbonate, except for 1A01's GlcNAc growth rate, which was $0.72 / \mathrm{hr}$, and 3B05's growth rate on acetate, which was $0.35 / \mathrm{hr}$. E. coli NCM3722 was grown at $37{ }^{\circ} \mathrm{C}$ in MOPS medium with $0.4 \mathrm{M} \mathrm{NaCl}$; the yields in $0.4 \mathrm{M} \mathrm{NaCl}$ were indistinguishable from those in $0.1 \mathrm{M} \mathrm{NaCl}$. Standard error estimated from at least three biological replicates. 

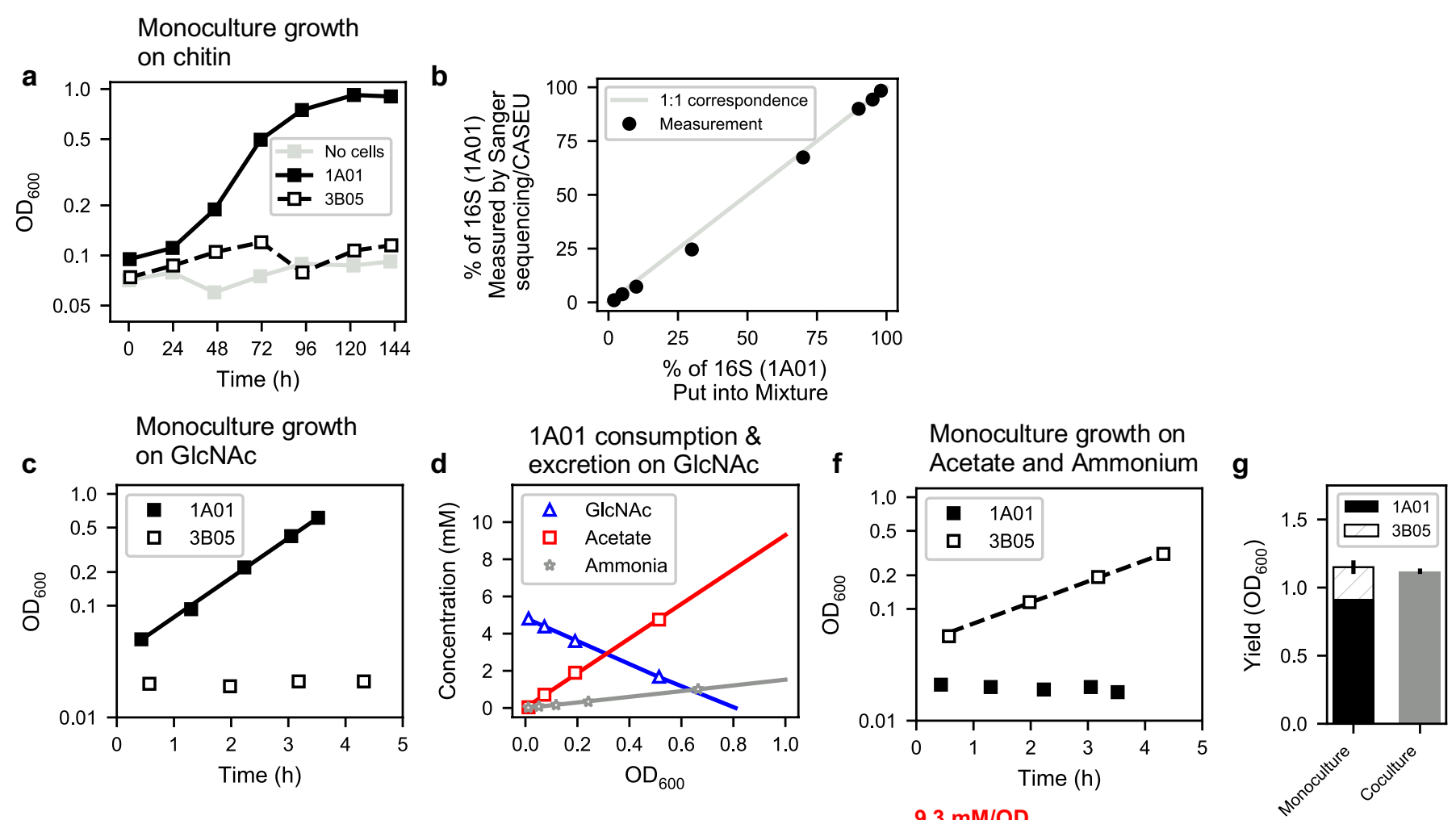

e
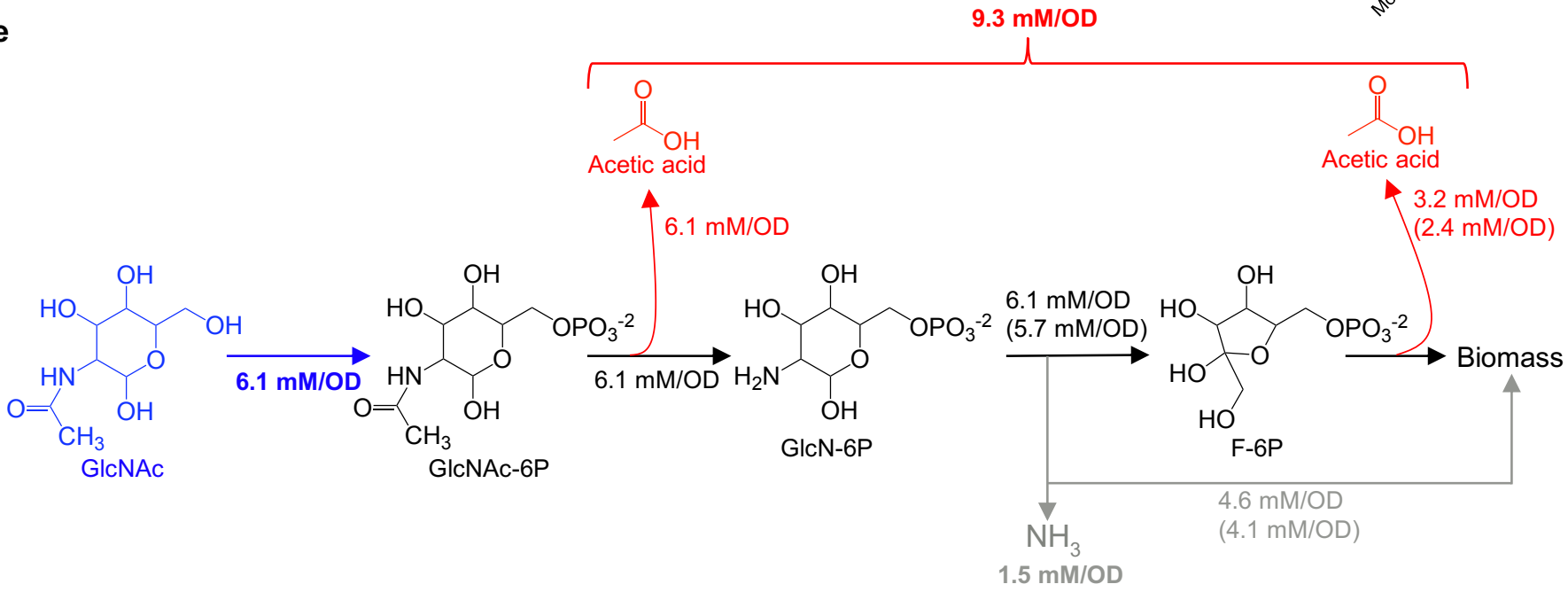

Extended Data Figure 1. Single-strain characteristics of 1A01 and 3B05. All results for batch culture growth described in this work were done in medium with $0.35 \mathrm{M} \mathrm{NaCl}$. The culture was maintained in water bath shaker at $27^{\circ} \mathrm{C}$ and $250 \mathrm{rpm}$. See details of growth medium and condition, including pre-culturing, in the Methods. (a) Growth on chitin. 1A01 and 3B05 were grown in monoculture with $0.2 \%$ w/v chitin chips as the sole carbon and nitrogen source. 1A01 was precultured in GlcNAc HEPES minimal medium with ammonium, and 3B05 was precultured in sodium acetate HEPES with ammonium. OD $_{600}$ measurements were taken from well-mixed culture after allowing the visible chitin chips to sink for 4 min. To see the effect of residual small chitin pieces in the culture, a 'no cells' control is also shown as light grey squares. (b) Accuracy of Sanger sequencing electropherograms in determining the composition of $1 \mathrm{~A} 01$ and 3B05 16S amplicon. Purified 16S amplicon of $1 \mathrm{~A} 01$ and 3B05 were mixed in defined proportions using their 
551 concentrations measured by Nanodrop (expected concentration, light grey line). The mixtures were submitted 552 for Sanger sequencing, and the resulting electropherograms were deconvolved using CASEU ${ }^{32}$ to determine 553 the fraction of each type of $16 \mathrm{~S}$ sequence. (c) Steady-state growth of 1A01 (filled black squares) and 3B05 554 (open black squares) on in minimal medium with $5 \mathrm{mM}$ GlcNAc as the sole carbon and nitrogen source 555 556 buffered by $40 \mathrm{mM}$ HEPES. See Methods for details of the growth protocol including preculturing preceding time ' 0 '. The solid line indicates an exponential fit to the $1 \mathrm{~A} 01$ growth curve $\left(0.81 / \mathrm{h}\right.$ with $\left.\mathrm{R}^{2}=0.999\right)$. (d) Depletion of GlcNAc (blue triangles) and accumulation of acetate (red squares) and ammonium (grey stars) during steady-state growth of $1 \mathrm{~A} 01$ on GlcNAc. The solid lines indicate linear fits, with the inverse biomass yield on GlcNAc being $6.1 \mathrm{mM} / \mathrm{OD}\left(\mathrm{R}^{2}=0.999\right)$, and acetate and ammonium excretion yields of 9.3 mM/OD $\left(\mathrm{R}^{2}=0.998\right)$ and $1.5 \mathrm{mM} / \mathrm{OD}\left(\mathrm{R}^{2}=0.999\right)$, respectively. The variance indicated in Extended Data Table 1 is from 3 biological replicates. (e) GlcNAc degradation pathway delineated previously for a Vibrio species ${ }^{33}$ and for $E$. coli ${ }^{63}$ depicting the excretion of acetic acid (red) and ammonium (grey). The yield numbers in bold are based on the measurements during exponential growth (panel d). The numbers in regular text are deduced based on stoichiometry and the measured values. The numbers in parenthesis are numbers obtained from similar measurements during exponential growth of $1 \mathrm{~A} 01$ on glucose and ammonium (where $5.7 \mathrm{mM}$ of glucose was consumed for $1 \mathrm{OD}$ of cells). We measured $9.3 \mathrm{mM} / \mathrm{OD}$ of acetate excreted during growth on GlcNAc. Based on stoichiometry, we expect $6.1 \mathrm{mM} / \mathrm{OD}$ of acetate from the conversion of GlcNAc-6-P to GlcN-6-P (left red arrow) and $3.2 \mathrm{mM} / \mathrm{OD}$ from acetate overflow ${ }^{55}$ (right red arrow). The latter is in line with what we measured for acetate excretion on glucose $(2.4 \mathrm{mM} / \mathrm{OD})$. Similarly based on stoichiometry, we expect $6.1 \mathrm{mM} / \mathrm{OD}$ of ammonium to be released from GlcN-P. We measured $1.5 \mathrm{mM} / \mathrm{OD}$ of ammonium excreted, implying $4.6 \mathrm{mM} / \mathrm{OD}$ of ammonium was assimilated into biomass, in line with the degree of ammonium assimilation we measured during growth on glucose $(4.1 \mathrm{mM} / \mathrm{OD})$. This analysis shows that growth yield on GlcNAc is similar to the yield on glucose and ammonium, so that the additional excretion of acetate and ammonium for the growth on GlcNAc compared to growth on glucose can be understood approximately as a result of the "extra" acetyl and amine groups accompanying the glucose in GlcNAc. In support of this interpretation, we found similar yield of acetate and ammonium due to excretion by E. coli growing on GlcNAc MOPS minimal medium with $0.4 \mathrm{mM} \mathrm{NaCl}$ (Extended Data Table 1). The excretion yield for glucose is also provided there for reference. (f) Steady-state growth of 1A01 (filled black squares) and 3B05 (open black squares) on HEPES-buffered minimal medium with $60 \mathrm{mM}$ sodium acetate as the sole carbon source and $10 \mathrm{mM}$ of ammonium chloride as the sole nitrogen source. The dashed black line indicates an exponential fit to the $3 \mathrm{~B} 05$ growth curve, with growth rate $0.43 / \mathrm{hr}\left(\mathrm{R}^{2}=0.999\right)$. The best-fit parameters in panels c, d, and f are summarized in Extended Data Table 1. (h) Solid left bar indicates the monoculture yield of 1A01 on $5 \mathrm{mM}$ GlcNAc. Open left bar indicates the monoculture yield of 3B05 on $7.4 \mathrm{mM}$ acetate; the latter is the amount of acetate excreted by $1 \mathrm{~A} 01$ after growth on $5 \mathrm{mM}$ GlcNAc). Grey right bar indicates the measured yield of the 1A01/3B05 coculture on $5 \mathrm{mM}$ GlcNAc. The two yields are approximately equal, indicating that cross-feeding in the coculture in strong buffer can be quantitatively understood in terms of the single-strain characteristics. 


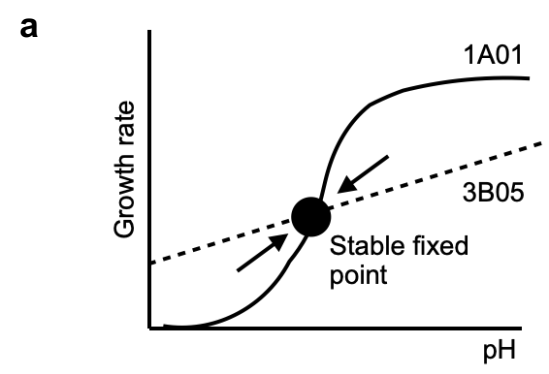

b

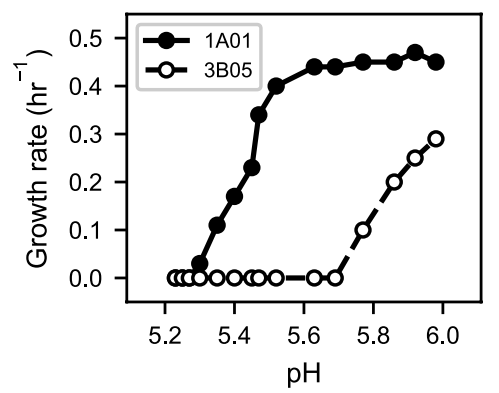

c

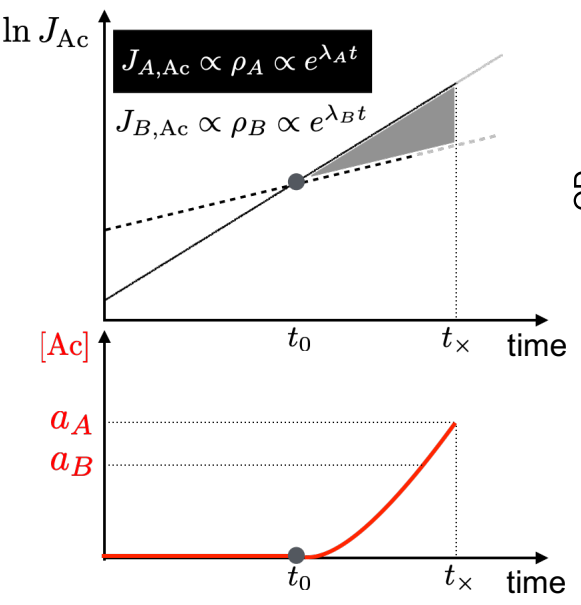

Coculture, Weak buffer,
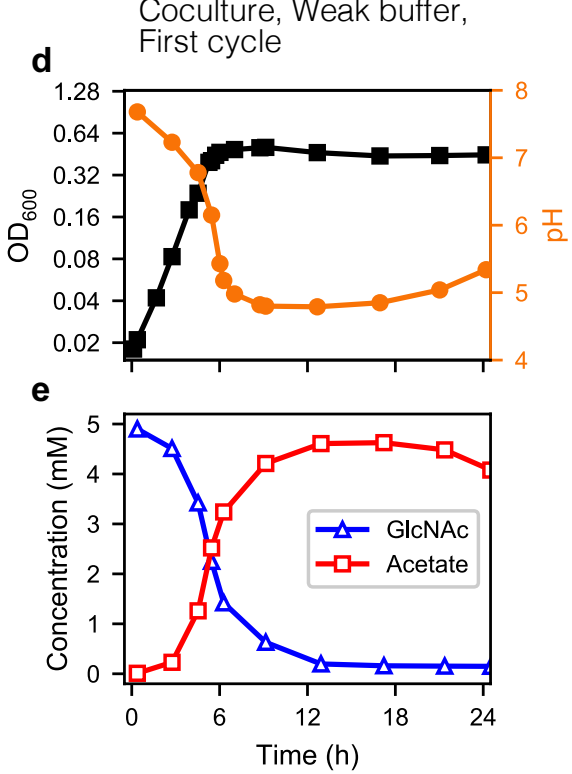

Extended Data Figure 2. Acetate cross-feeding cannot support exponential growth of 1A01 and 3B05 coculture. (a) In the canonical scenario of syntrophy, growth of the strain generating toxicity should be more sensitive to toxicity (solid black line) than the strain relieving toxicity (dashed black line) as illustrated. The intersection of these two lines is the stable fixed point describing a stable, exponential growing coculture: above this $\mathrm{pH}$, the faster-growing strain (1A01) would excrete more acetate than what the de-toxifier (3B05) could take up, resulting in a decrease in the $\mathrm{pH}$. Below this $\mathrm{pH}, 3 \mathrm{~B} 05$ would be able to take up acetate faster than 1A01 could produce it, resulting in an increase in the $\mathrm{pH}$. This balance would lead to a stable exponential growth phase for the coculture. (b) Dependences of the growth rate of 1A01 (solid circles) and 3B05 (open circles) on the medium $\mathrm{pH}$. Cells were grown in minimal medium buffered by $10 \mathrm{mM}$ MES with different ratios of the acid and base form to obtain the desired $\mathrm{pH}$; otherwise, the medium was identical to the HEPESbuffered medium. Glycerol was used as the sole carbon source as both strains grew on it and neither strain excreted acetate or other fermentation products which would have changed the medium $\mathrm{pH}$ and make this experiment difficult to do. The data shows that $3 \mathrm{~B} 05$ is more sensitive to $\mathrm{pH}$ than $1 \mathrm{~A} 01$, hence precluding the scenario of stable exponentially growing coculture depicted in panel a. (c) Qualitative account of the coculture dynamics of $1 \mathrm{~A} 01$ and $3 \mathrm{~B} 05$ in weak buffer. Because the acetate excretion $\left(J_{A, \mathrm{Ac}}\right)$ and uptake flux $\left(J_{B, A c}\right)$ is proportional to the density of $1 \mathrm{~A} 01$ and $3 \mathrm{~B} 05$, respectively (Eq. (1.4), Supp. Note 1), each flux increases exponentially with the corresponding growth rate $\left(\lambda_{A}\right.$ and $\left.\lambda_{B}\right)$ as show by the solid and dashed line. Since $\lambda_{A}>\lambda_{B}$ at all pHs (panel b), acetate excretion by $1 \mathrm{~A} 01$ (solid black line) will eventually exceed its consumption by 3B05 (dashed black line); this is marked by the circle at time $t_{0}$. For $t>t_{0}$, the acetate concentration would rise approximately exponentially in time, until 3B05 stops growing when the stopping concentration $a_{B}$ is reached. Soon after that, acetate concentration reaches $a_{A}$ at some time $t_{\mathrm{x}}$, when $1 \mathrm{~A} 01$ ceases growing also. (d) and (e), Measurement during the first 24-hr growth period of the coculture in weaklybuffered medium. (d) shows the OD (black squares) and $\mathrm{pH}$ (orange circles) and (e) shows the GlcNAc (blue triangles) and Acetate (red squares) concentrations. 

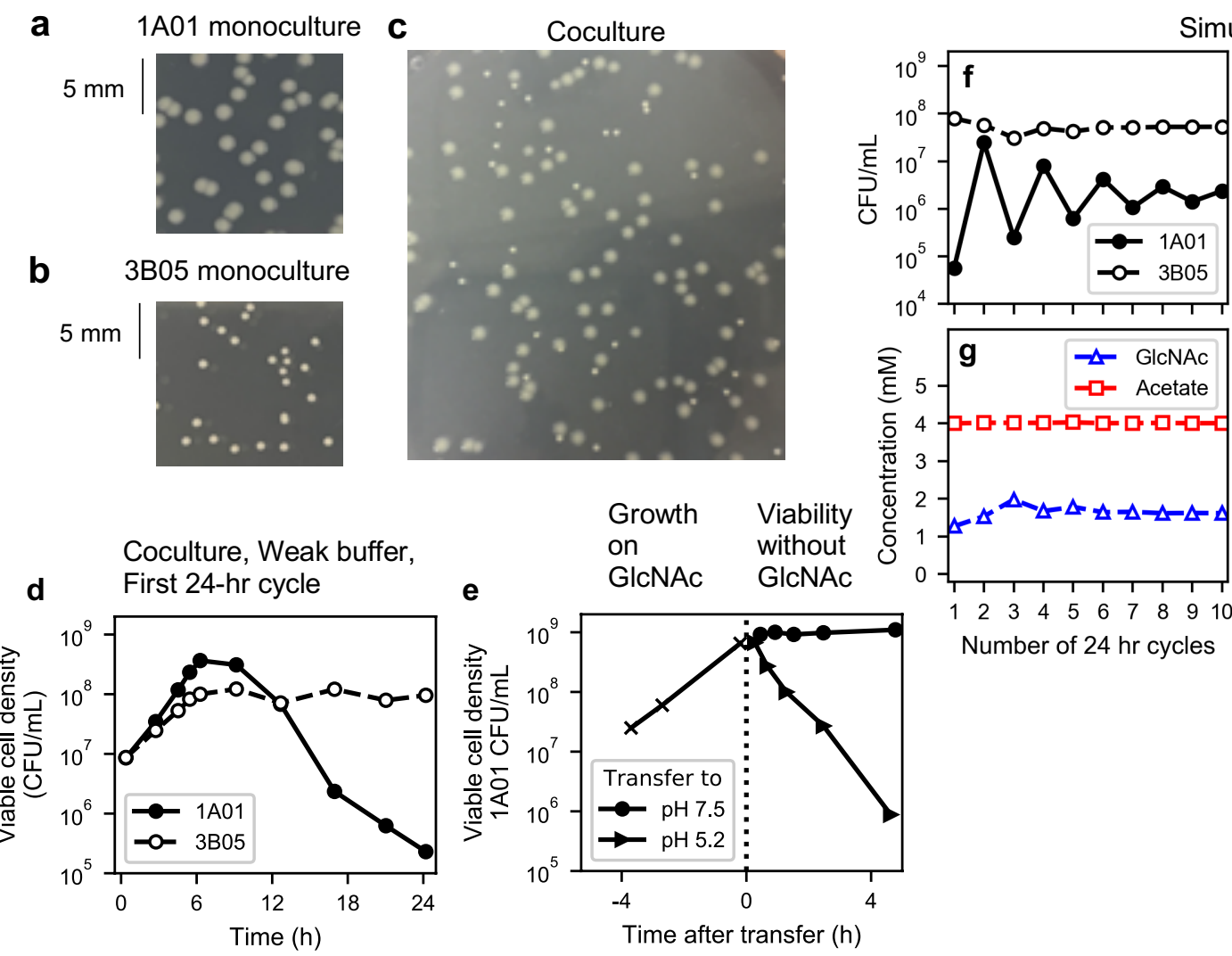

Simulation
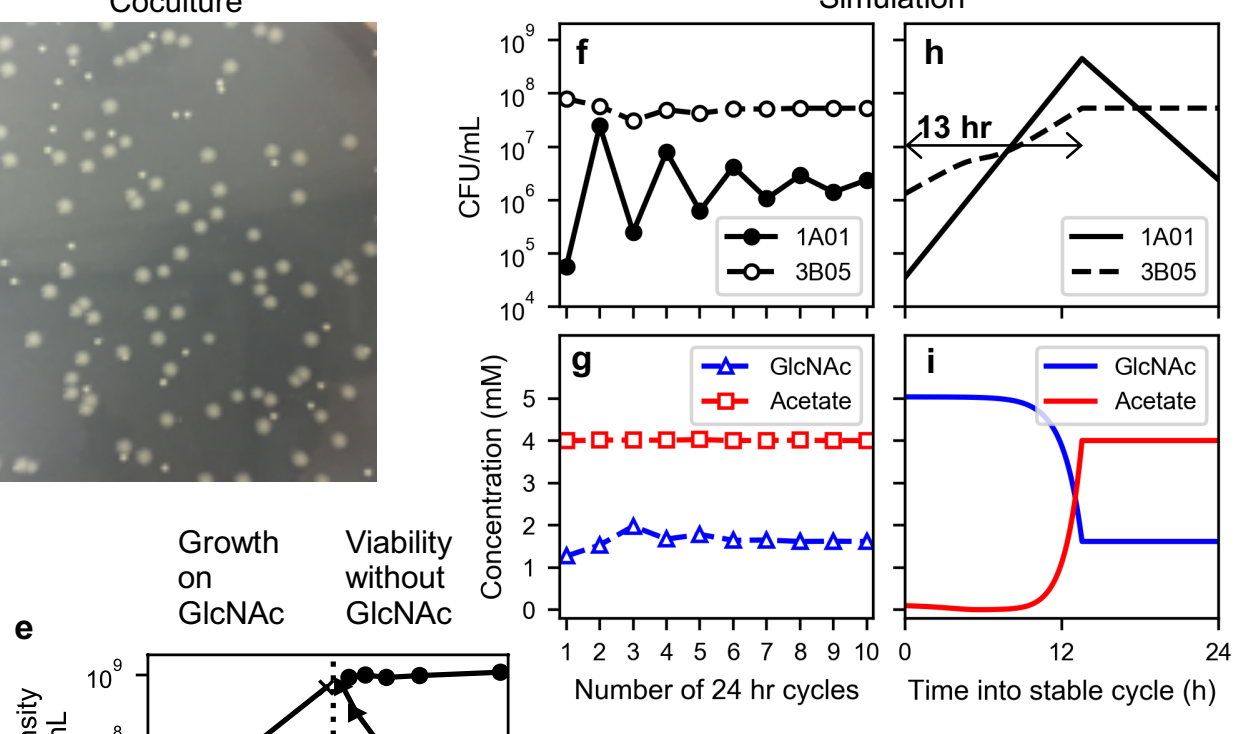

617

618

619

620

621

622

623

624

625

626

627

628

629

630

631

632

633

634

635

636

637

638

639

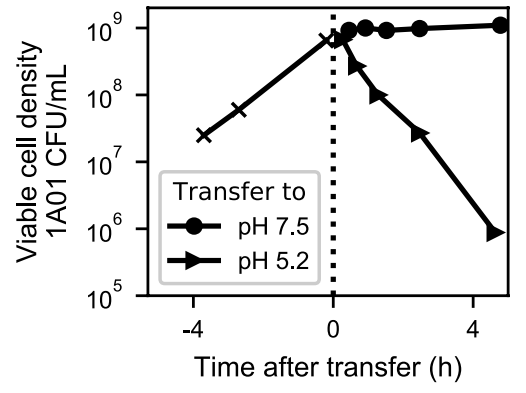

ime into stable cycle (h)

Extended Data Figure 3. 1A01 loses viability in acidified medium due to acetate excretion. (a-c) Colonies of 1A01 and 3B05 exhibit different morphologies. 1A01 monoculture (a), 3B05 monoculture (b), and $1 \mathrm{~A} 01 / 3 \mathrm{~B} 05$ (c) coculture growing in HEPES buffered medium was plated on $1.5 \%$ agar with rich medium (marine broth). After incubation of the plate for $\sim 36 \mathrm{hr}$ at $27^{\circ} \mathrm{C}, 1 \mathrm{~A} 01$ and $3 \mathrm{~B} 05$ colonies were visibly different. 1A01 colonies have a much larger area and an off-white color, while 3B05 colonies are punctate and white. (d) Viable cell counts for 1A01 (filled black circles) and 3B05 (open black circles) measured during the first growth period of the coculture grown in weak buffer. (e) The death of 1A01 in monoculture was characterized by measuring the viable cell density (colony forming units or CFU per $\mathrm{mL}$ of culture) before and after exponentially growing $1 \mathrm{~A} 01$ monoculture is transferred to medium with different $\mathrm{pH}$ : Before the transfer, 1A01 grew in HEPES-buffered medium with GlcNAc as the sole carbon and nitrogen source. Viable cell count (black x's) showed exponential growth consistent with growth rate obtained from OD measurement (Extended Data Fig. 1c). At $\mathrm{OD}_{600}$, indicated as time $\mathrm{t}=0$ (black dotted line), these cells were washed and resuspended into GlcNAc-free medium with $2 \mathrm{mM}$ bicarbonate buffer, with the $\mathrm{pH}$ set to 7.5 (black circles) or $\mathrm{pH} 5.2$ (black triangles). In the latter case, $3.6 \mathrm{mM}$ of acetic acid was added to lower the $\mathrm{pH}$. Rapid drop of viable 1 A01 cells was observed for the ones transferred to the medium at low $\mathrm{pH}$ but not at normal pH. (f-i) Simulation result using a model of 1A01 and 3B05 cross-feeding that includes cell death (Supp. Note 2), applied to describe the dynamics of the coculture in 24-h growth-dilution experiments with $40 \mathrm{x}$ dilution. When the acetate reached $4 \mathrm{mM}, 1 \mathrm{~A} 01$ cells decreased with a death rate $0.5 / \mathrm{hr}$ as determined in panel (d). The density of live 1A01 cells is indicated by a solid black line and 3B05 by a dashed black line. The concentrations of acetate and GlcNAc are indicated by the red and blue lines, respectively. (f) and (g) show the simulation results at the end of each 24-h cycle; (h) and (i) show the simulation results during Cycle 10 , after the coculture has stabilized. 
640 The simulation results show that the model with cell death is able to produce the co-existence of $1 \mathrm{~A} 01$ and 641 3B05. The effect of cell death can be understood as follows: since the growth rate for 3B05 on acetate in the 642 weakly-buffered medium is $0.35 \mathrm{hr}^{-1}$, it needs at least $11.8 \mathrm{hr}$ to grow $40 \mathrm{x}$ to make up for the dilution at the 643 end of a cycle. The rapid death of $1 \mathrm{~A} 01$ means that, following dilution, it needs to grow $\gg 40 \mathrm{x}$ to maintain 644 its population. Thus, 1A01 must undergo additional doublings until it reaches densities that can acidify the 645 culture. This delays acetate buildup and the ensuing stoppage of coculture growth, thereby giving 3B05 more 646 time to replicate and catch up for the 40x density increase required for coexistence. Quantitatively, our model 647 shows that coexistence can be achieved in a stable cycle with growth occurring for the first $\sim 13 \mathrm{~h}$ (panels $\mathrm{h}$ 648 and i), as opposed to the first cycle where growth was limited to the first 6 hours (panel d). This results in a 649 reduced period of growth arrest and hence a reduced drop in the viable 1A01 cells, such that in one cycle 650 1A01 and 3B05 each have just enough time to increase its density 40x. Thus, the death of a faster species 651 can effectively delay the onset of toxicity, thereby enabling a slower cross-feeder to avoid extinction.

652

In this model, the key to the coculture finding a stable cycle is that it stops growing prematurely, i.e., before 654 the full consumption of GlcNAc. This point can be adjusted dynamically until a stable cycle is reached where 655 the growth period for $1 \mathrm{~A} 01$ is just enough to compensate for its death and the dilution factor. However, our data on GlcNAc and acetate at the end of each cycle of the 24-h growth-dilution experiment in weak buffer (Fig. 1d) showed major differences from the simulation result here: More acetate and less GlcNAc than predicted were found at the end of Cycle 1, and, once the coculture stabilized, GlcNAc and acetate were entirely consumed. Also, the ratio of viable counts of 1A01 is comparable to 3B05 (Fig. 1f) whereas it is almost 100x lower than 3B05 in this model (panel f). Therefore, death of 1A01 was not the cause of stable 661 coexistence observed in weak buffer.

662 
665

666

667

668

669

670

671

672

673

674

675

676

677

678

679

680 a

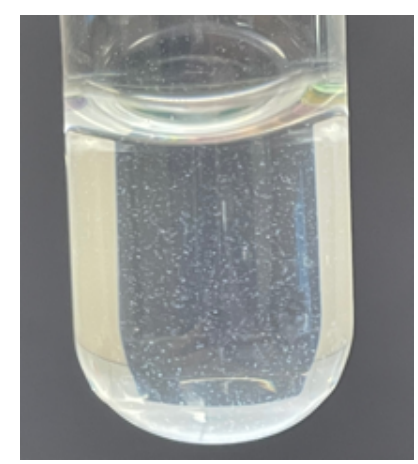

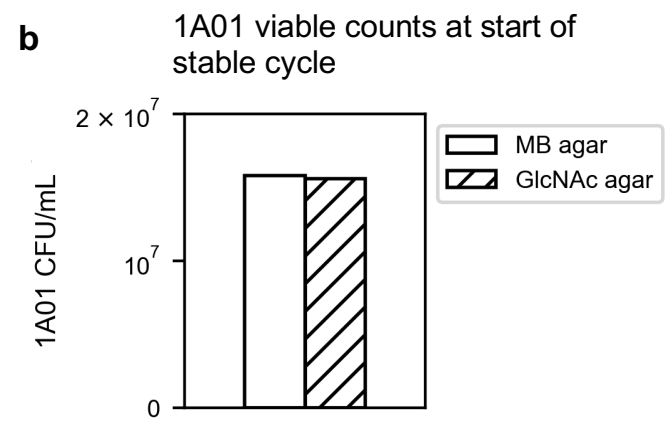

Extended Data Figure 4. 1A01 aggregates during lag phase of the stable cycle. The drop of 1A01 colony count during the first 6 hours after dilution in the stable cycle (Fig. 2a) is not due to the loss of viability but due to cell aggregation. (a) Picture of coculture taken during the lag phase of the stable cycle of the 24-h growth/dilution experiment in weak buffer, 3 hours after diluting into fresh GlcNAc medium: Macroscopic aggregates are seen in the culture. Thus, aggregation of 1A01 cells may account for all or a part of the drop in 1A01 colony count observed during the lag phase. (b) To see whether a fraction of stationary 1A01 cells may nevertheless die from the previous cycle after transferring to fresh GlcNAc medium, 1A01 cells were plated onto GlcNAc minimal medium plate and marine broth plate immediately following dilution of the stable cycle. On either plate, individual cells have no chance to aggregate. If exposure to GlcNAc minimal medium was somehow toxic to $1 \mathrm{~A} 01$, we expect to see a drop in colony count on the GlcNAc minimal medium plate compared to marine broth plate, since the latter culture grew rapidly in marine broth without lag (filled squares, Fig. 4d). However, we saw no difference in colony count between the two plate types. Thus, there is no death of $1 \mathrm{~A} 01$ due to dilution into GlcNAc minimal medium. 


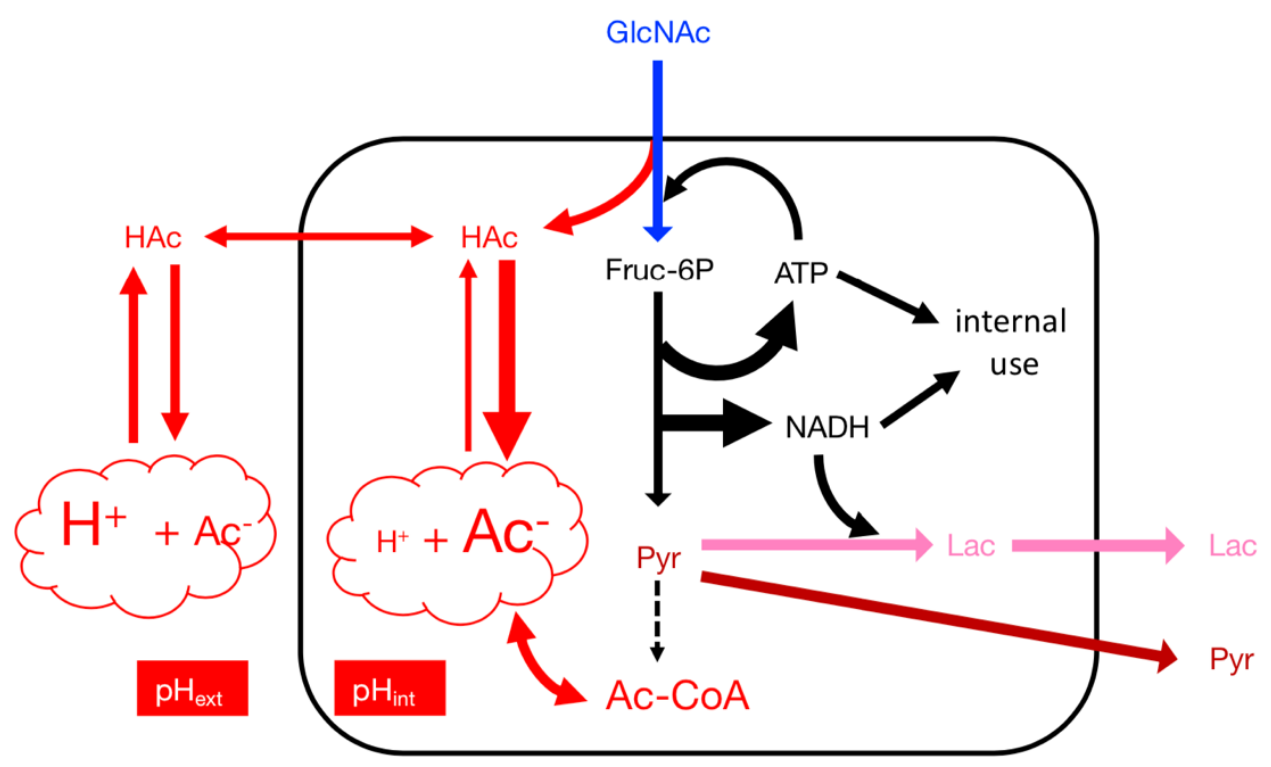

681

682

683

684

685

686

687

688

689

690

691

692

693

694

695

696

697

698

699

700

701

702

703

704

705

706
Extended Data Figure 5. Metabolism under acetate stress. The basic physiological problem bacterial cells face under "acetate stress", outlined by J. Russell decades ago ${ }^{41}$, is illustrated by the red part of the cartoon above: Acetic acid (HAc) is in equilibrium with the anion species, Acetate $\left(\mathrm{Ac}^{-}\right)$, with the ratio of the two concentrations governed by the $\mathrm{pH}$, i.e., $\left[A c^{-}\right]=[H A c] \times 10^{p H-p K_{a}}$ where $p K_{a} \approx 4.75$. Because HAc, a small, neutral molecule is permeable through the cell membrane, the ratio of the intracellular and extracellular acetate concentrations is given by

$$
\frac{\left[A c^{-}\right]_{\text {int }}}{\left[A c^{-}\right]_{\text {ext }}}=10^{p H_{\text {int }}-p H_{\text {ext }}}
$$

assuming the $\mathrm{pK}_{\mathrm{a}}$ value does not change significantly in the cytoplasm. When the medium $\mathrm{pH}$ drops to $\sim 5$, and assuming internal $\mathrm{pH}$ is maintained at $\sim 7$, then the internal acetate concentration would be $\sim 100 \mathrm{x}$ larger than the external concentration. For external concentration of $\sim 3 \mathrm{mM}$ as encountered in this study, the internal concentration would be in the range of $\sim 300 \mathrm{mM}$, which would be of the order of the sum of the concentrations of all intracellular metabolites ${ }^{43}$. Osmotic balance would force a substantial part of the intracellular metabolites to be released from the cell, since the internal acetate concentration is fixed by the extracellular acetate concentration. The excretion of internal metabolites was indeed detected for $E$. coli cells subjected to acetic acid shock ${ }^{42}$.

Aside from this osmotic imposed excretion effect, we expect the uptake of GlcNAc in the medium to give rise to additional excretion of internal metabolites as illustrated in the sketch above: glycolysis would bring the carbon flux down to pyruvate, but we expect decarboxylation of pyruvate to be "plugged" due to a very high Ac-coA pool (or a lack of free coA pool). In this case, the build-up of the internal pyruvate would leak out of the cell as pyruvate or its derivatives, e.g., lactate. In this process, GlcNAc is converted into Acetate, Pyruvate and Lactate (and ammonium, not shown), with the cell gaining 1 NADH and 2 ATP per GlcNAc molecule for cellular maintenance use. 

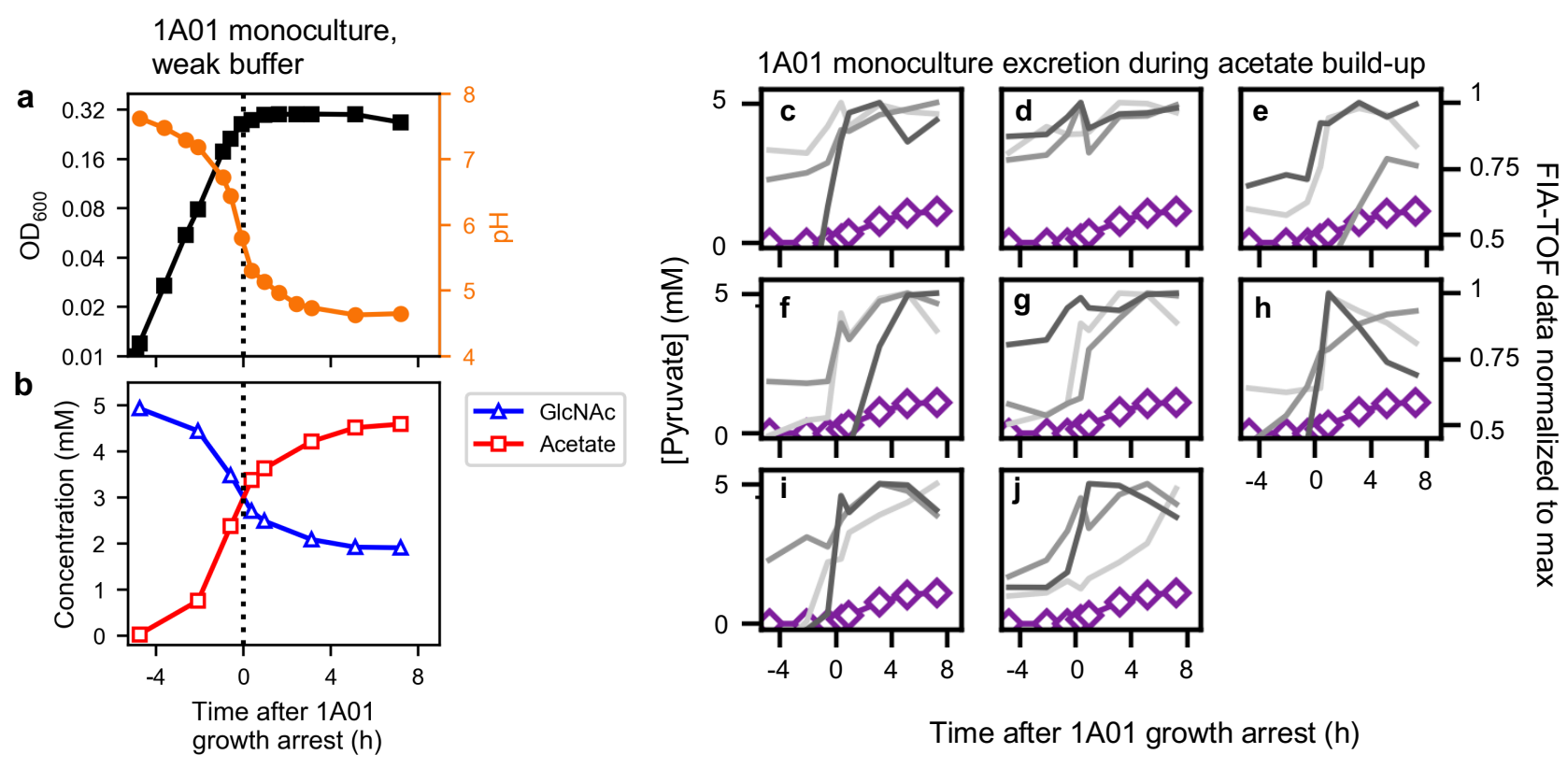

\begin{tabular}{|c|c|c|c|}
\hline \multirow[b]{2}{*}{ Panel } & \multicolumn{3}{|c|}{ Legend } \\
\hline & 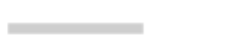 & 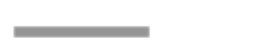 & 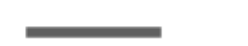 \\
\hline c & 2-deoxyribose & 2-hydroyglutarate & glycerate \\
\hline d & 2-aceto-lactate & lactate & 2-ketoglutarate \\
\hline e & 1-amino.. & 3-dihydroshikamate & 3,4-hydroxy... \\
\hline f & 4-amino-butyrate & Coum.. & 4-hydroxybenzoate \\
\hline g & 5-oxoproline & Cis-aconitate & 2-methylmaleate \\
\hline h & Ribulose-5p & glycine & ascorbate \\
\hline $\mathbf{i}$ & Aspartate-semi & Glutamate-semi & glutamate \\
\hline j & GIcNAc-6P & Acetyl-glutamate & pyruvate \\
\hline $\begin{array}{l}\text { 1-amin } \\
\text { 3,4-hyc } \\
\text { Coum. } \\
\text { Semi = }\end{array}$ & $\begin{array}{l}\text { amino-propan-2-on } \\
=3,4 \text {-hydroxyphen } \\
\text { Imaraldehyde } \\
\text { dehyde }\end{array}$ & & \\
\hline
\end{tabular}

\section{Extended Data Figure 6. Excretion of metabolites in 1A01 monoculture during growth on GlcNAc in} weak buffer. 1A01 was grown in $5 \mathrm{mM}$ GlcNAc in the weak $2 \mathrm{mM}$ bicarbonate buffered medium. (a) shows OD (squares) and $\mathrm{pH}$ (circles), and (b) shows the GlcNAc and acetate concentrations in the medium. A black dotted line indicates the point at which 1A01 stops growing. (c-j) FIA-QTOF-MS was applied to the same spent media samples measured in part $b$. The right $y$-axis indicates the measured intensity normalized by the max intensity during the time course for that metabolite. For reference, we additionally plotted the absolute concentration of pyruvate (purple diamonds) independently measured on the same samples using HPLC (left y-axis). 

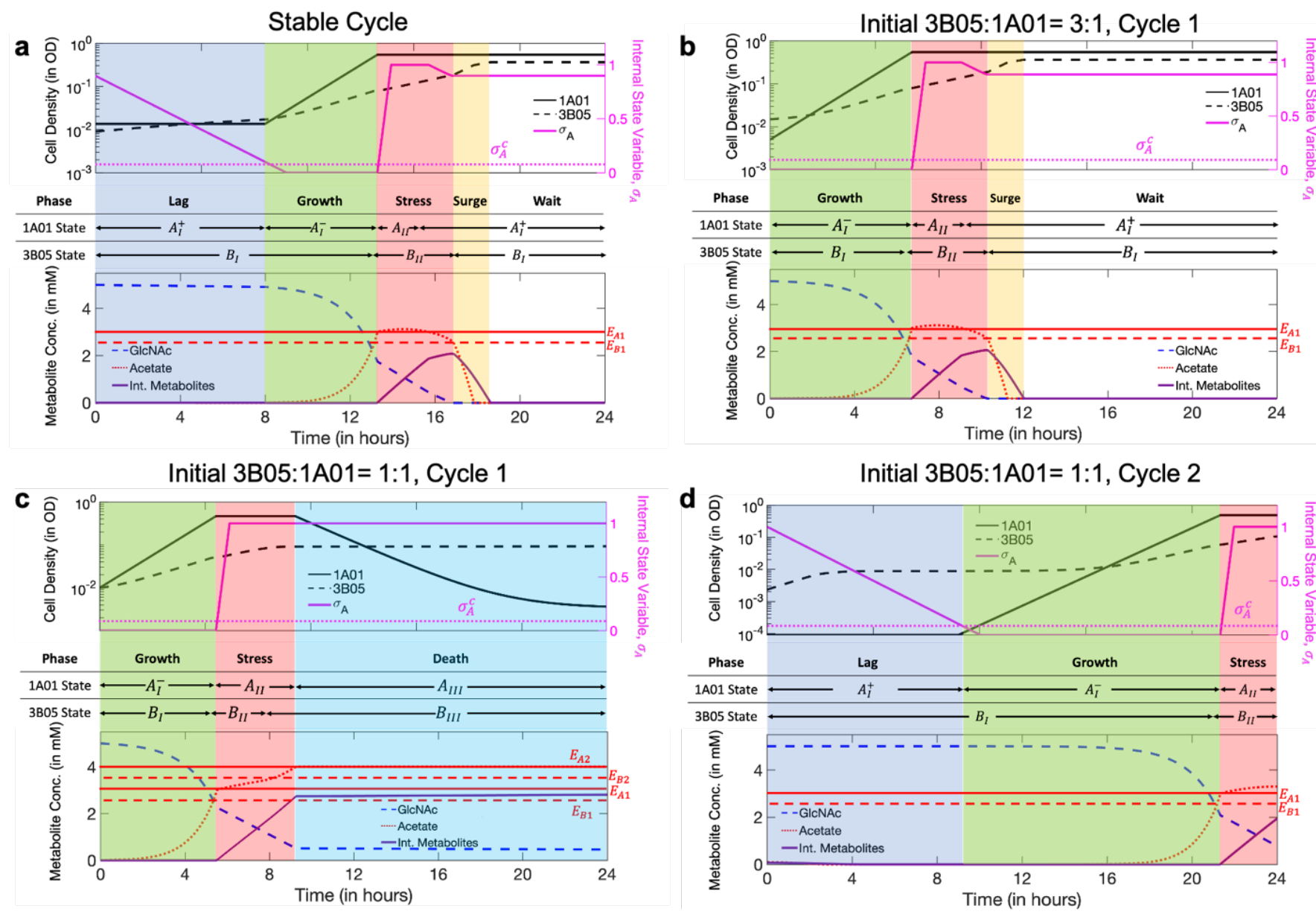

Extended Data Figure 7. Dynamics of the Stable Cycle and its approach from initial conditions. Our model of dynamic cross-feeding, as defined by Eqs. (3.1)-(3.5) and (3.16) in Supp Note 3 (with the rate functions given by Table N3.1 and N3.2, and the parameters given by Table N3.3), are studied by numerical simulation. Outputs of the model are shown in Fig. 5 and Extended Data Fig. 8 for two initial strain density ratios 3B05:1A01=1:1 and 3:1. We describe here the dynamics of the stable cycle and the transient dynamics for each initial ratio leading to the stable cycle. We also illustrate the dynamics of the internal variable $\sigma_{A}$ used in the model to capture the effect of internal metabolite depletion on growth recovery by 1 A01 (Supp Note 3).

(a) We first describe the stable cycle. Starting from $\sim 8$ hours into the cycle, the system is in the "Growth Phase" (shaded green region) where both 1A01 and 3B05 grow at their normal rates (solid and dashed black lines, respectively, top plot), as there is abundant GlcNAc (dashed blue line, bottom plot); the pH is in the normal range (acetate, dotted red line bottom plot) below the threshold values indicated by the red horizontal lines above which growth stops; and the internal state of $1 \mathrm{~A} 01\left(\sigma_{A}\right.$, solid magenta line in top plot $)$ is below the threshold value ( $\sigma_{A}^{c}$, dotted horizontal magenta line) above which growth stops. In this phase, $1 \mathrm{~A} 01$ cells are in state $A_{I}^{-}$and 3B05 cells are in state $B_{I}$ (as defined in Fig. 5a). As 1A01 grows rapidly in this phase, acetate quickly accumulates in the coculture. This leads to the growth arrest of $1 \mathrm{~A} 01$ (entering state $A_{I I}$ ) when the acetate concentration exceeds the threshold $E_{A 1}$. In this state, $1 \mathrm{~A} 01$ continues to consume GlcNAc, turning it into internal metabolites which are then excreted. Additionally, the internal variable $\sigma_{A}$ increases rapidly, reflecting the loss of other internal metabolites due to acetate accumulation (Fig. 4). This phase is 
labelled as the "Stress Phase" and shaded pink. Here 3B05 is in state $B_{I I}$ and continues to grow, albeit slowly, assisted by the internal metabolites excreted from 1A01. The growth of 3B05 slowly removes acetate from the medium. When the acetate concentration falls below the threshold $E_{B 1}, 3 \mathrm{~B} 05$ is back in state $B_{I}$ and can resume normal growth. It actually grows even faster than in the Growth Phase due to the presence of internal metabolites which boosts its growth. This phase is thus called the "Surge Phase" and is shaded as yellow. $1 \mathrm{~A} 01$ is no longer inhibited by acetate either in this phase. But it cannot grow for two reasons: First, GlcNAc is depleted, and second, its internal variable $\sigma_{A}$ is large. It is in state $A_{I}^{+}$. Since acetate and internal metabolites are no longer produced by $1 \mathrm{~A} 01$ in this phase (as GlcNAc is depleted), while 3B05 continues to consume them, eventually, 3B05 clears the acetate and stops growing. The coculture stagnates at this point and enters the "Wait Phase" (not shaded). It would remain in this phase until dilution into fresh medium in the new cycle. With the infusion of fresh GlcNAc, 1 A01 starts its slow recovery by reducing the internal variable $\sigma_{A}$ while consuming GlcNAc, mimicking the replenishment of the depleted internal metabolites inside the cell. $1 \mathrm{~A} 01$ would remain in state $A_{I}^{+}$as long as $\sigma_{A}>\sigma_{A}^{c}$ ("Lag Phase", purple shade). During this time, 1A01 continues to convert GlcNAc into acetate and internal metabolites, which support the growth of 3B05 (in state $B_{I}$ ). When $1 \mathrm{~A} 01$ is finally able to grow again (when $\sigma_{A}$ drops below the threshold $\sigma_{A}^{c} \sim 8$ hours later), the system enters the Growth Phase again and the cycle repeats.

(b) For the 3:1 initial ratio, in the first cycle, both 1A01 and 3B05 grow at their normal rates from time 0, when there is abundant GlcNAc and normal pH ("Growth Phase", green shaded region). This leads to acetate accumulation and stoppage of growth of 1A01 ("Stress Phase", pink shaded region) as described for the stable cycle in Panel a. The internal variable $\sigma_{A}$ also increases rapidly, indicating a loss of internal metabolites. The high initial ratio of 3B05 to $1 \mathrm{~A} 01$ ensures that there is enough 3B05 to clear the acetate excreted by 1A01, until the point when GlcNAc is almost depleted, after which the coculture stagnates (Wait Phase, unshaded). Because the first cycle does not have a Lag Phase (as in the stable cycle shown in Panel a), the coculture has a prolonged Wait Phase. Starting from the second cycle, the Lag Phase occupies the first $\sim 8$ hours of the cycle, putting the coculture in the stable cycle.

(c) For the 1:1 initial ratio of 1A01 and 3B05 (Fig. 5), in the first cycle, 1A01 and 3B05 grow when there is abundant GlcNAc, and normal $\mathrm{pH}$ as described in panels a and b ("Growth Phase', green shaded region). However, the lower initial density of 3B05 makes it unable to clear acetate rapidly, so that 1A01 enters growth arrest with a lot of GlcNAc still remaining ("Stress Phase", pink shaded region). In the Stress Phase, 1A01 continues to consume GlcNAc and excrete acetate and internal metabolites. Because 3B05 grows slowly and hence consumes acetate slowly in the Stress Phase, acetate continues to accumulate, such that the system is pushed into very high acetate concentrations where $3 \mathrm{~B} 05$ is in state $B_{I I I}$ and cannot grow, and $1 \mathrm{~A} 01$ is in state $A_{I I I}$ and dies ("Death Phase", cyan shade). In this phase, the density of 1A01 drops rapidly. The cycle also ends with a very high value of the internal variable $\sigma_{A}$ indicating a severe loss of internal metabolites.

(d) At the start of the second cycle for a 1:1 initial ratio of 1A01 and 3B05 (Fig. 5), 1A01 stops dying as acetate is cleared out by dilution. However, due to the large value of $\sigma_{A}$ going into the cycle, $1 \mathrm{~A} 01$ remains in state $A_{I}^{+}$while its internal state recovers as long as $\sigma_{A}>\sigma_{A}^{c}$, while 3B05 grows on the lingering acetate from the previous cycle and the internal metabolites excreted by $1 \mathrm{~A} 01$ as $1 \mathrm{~A} 01$ is in state $A_{I}^{+}$("Lag Phase", purple shade). Once $\sigma_{A}<\sigma_{A}^{c}$, both 1A01 and 3B05 grow exponentially ("Growth Phase", green shade). This Growth Phase lasts much longer than the green phases described in Panels a-c as the starting density of 1A01 is very low, and thus it takes a long time for acetate to accumulate. 21 hours after the start of the cycle, acetate accumulates enough to stop the growth of 1A01 and 3B05 ("Stress Phase", pink shade). Due to the 
786 prolonged period at very low densities, 1A01 is unable to consume all of the GlcNAc by the end of the cycle. 787 Further, because of the short duration in Stress Phase in this cycle, 3B05 is unable to grow and clear the 788 acetate accumulated during the Growth Phase by the end of the cycle. However, the stress at the end of $2^{\text {nd }}$ 789 cycle does lead to a high value of $\sigma_{A}$ and a significant Lag Phase in the subsequent $\left(3^{\text {rd }}\right)$ cycle, placing the 790 coculture in the stable cycle.

792 From the detailed analysis of the above two examples, we see that if the initial ratio of 3B05 to $1 \mathrm{~A} 01$ is high 793 enough, it will enter a stable cycle provided that the duration of the cycle is long enough to contain the four 794 crucial phases: Lag, Growth, Stress, and Surge. If the initial ratio is lower, then the coculture may be able to 795 recover after 1 A01 suffers death for a while. 

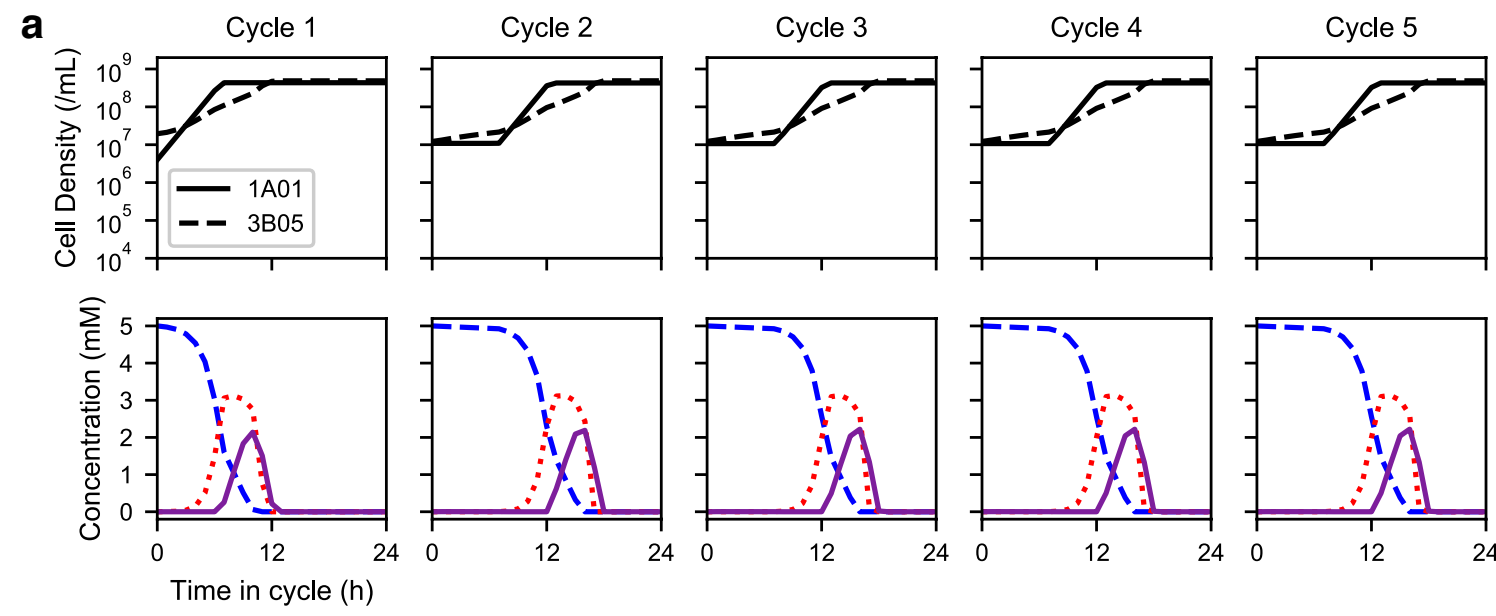

b
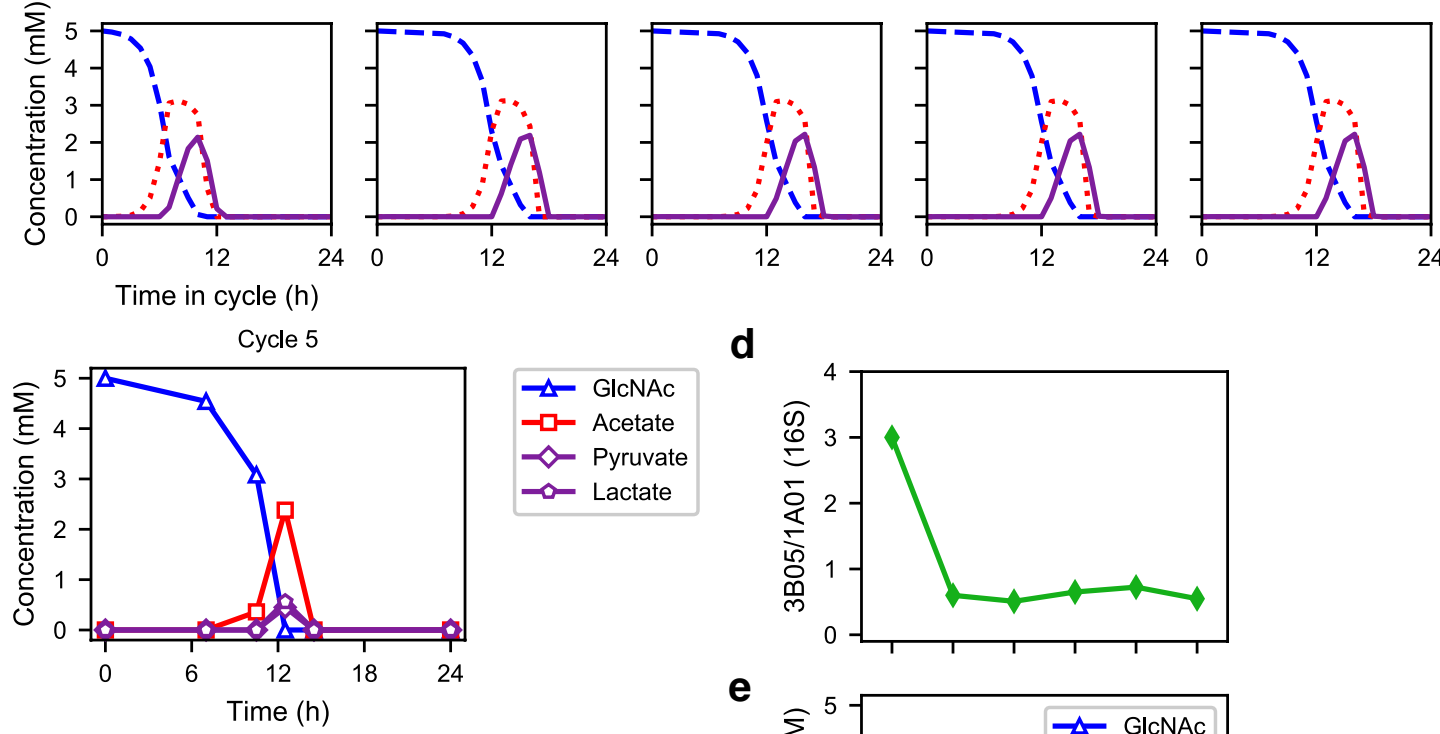

- - GlcNAc

\#.. Acetate

Int Met

d
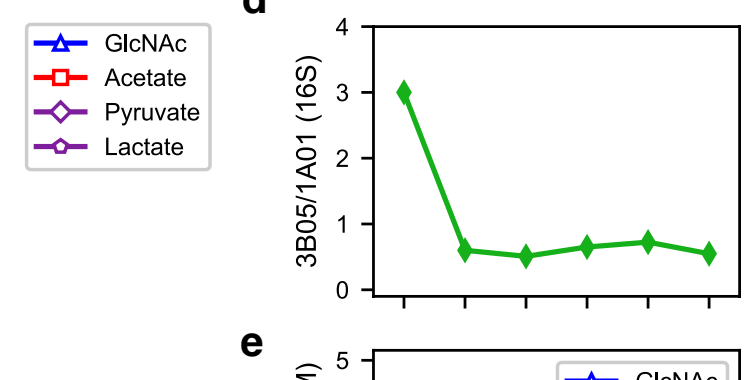

e
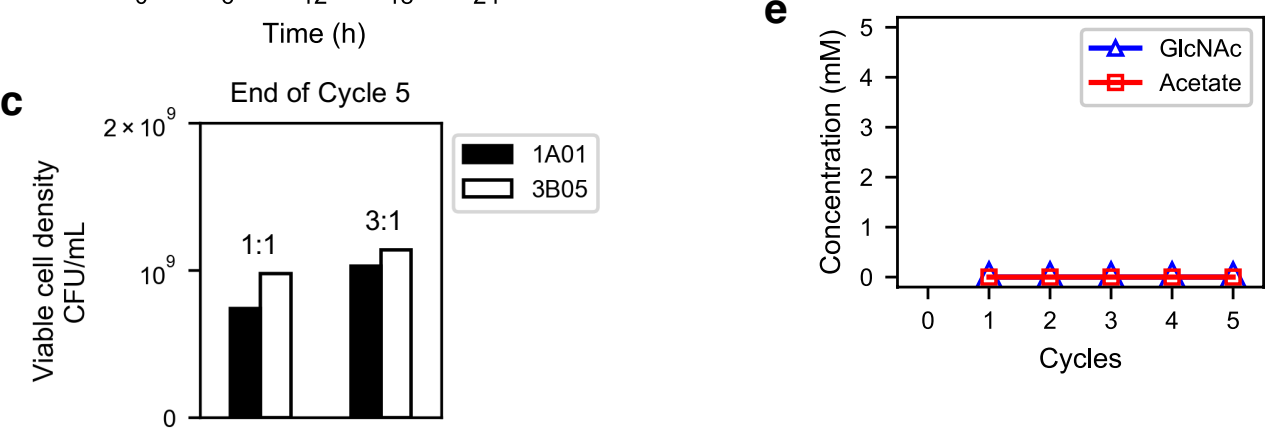

Extended Data Figure 8. Coexistence of coculture with a 3:1 initial ratio of 3B05 to 1A01. (a) Using the model of 1A01/3B05 cross-feeding dynamics described in Supp Note 3, we simulated the coculture in 24-h growth/dilution cycle starting with a 3:1 ratio of 3B05 to $1 \mathrm{~A} 01$. The result shows that the stable cycle is attained after a single 24-h cycle, compared to the 3 cycles needed to reach the stable cycle when starting with 1:1 initial ratio (Fig. 5b). The dynamics of the metabolites (GlcNAc, dashed line, acetate, dotted line, internal metabolites, solid line) during the stable cycle are expected to be the same as that obtained for the 1:1 initial ratio (Fig. 5b). See Extended Data Fig. $7 \mathbf{b}$ for a description of the approach to the stable cycle. These predictions were tested experimentally in panels (b)-(e) with experimental parameters identical to those described in the main text, i.e., for 1A01/3B05 growing in minimal medium with $5 \mathrm{mM}$ GlcNAc as the sole carbon and nitrogen source, buffered by $2 \mathrm{mM}$ sodium bicarbonate, with 24-h growth/dilution cycles and 40x dilution, except for a 3:1 starting ratio, with total initial $\mathrm{OD}=0.02$. (b) The concentrations of GlcNAc, acetate, pyruvate (open purple diamonds), and lactate (open purple pentagons) on day 5 of a growth/dilution experiment started with a 3:1 ratio of 3B05 to 1A01. The same acetate peak appeared transiently, at around $\mathrm{t}=12 \mathrm{~h}$ where GlcNAc was depleted, as seen in the stable cycle starting from 1:1 initial ratio (Fig. 2b). (c) Viable cell counts of 1A01 and 3B05 measured at the end of cycle 5 for the 1:1 and 3:1 
813 starting ratios, showing that the final species abundance ratio was approximately 1:1 as expected. (d) Ratio 814 of $16 \mathrm{~S}$ reads (3B05:1A01), and (e) [GlcNAc] (open blue triangles) and [Acetate] (open red squares) were 815 measured at the end of each growth cycle. The data support the rapid approach to the stable cycle as predicted. 816 Thus, the same stable cycle is reached for both initial ratios of 3B05 to 1A01, despite very different transient 817 dynamics. 